Pacific Journal of Mathematics

INVARIANT HARMONIC ANALYSIS ON SPLIT RANK ON
GROUPS WITH APPLICATIONS 


\section{INVARIANT HARMONIC ANALYSIS ON SPLIT RANK ONE GROUPS WITH APPLICATIONS}

\section{P. C. Trombi}

Let $G$ be a real connected noncompact semisimple Lie group with finite center; we shall denote the algebras of Lie groups $L$ by the corresponding lower case German letter, l. We assume that if $G_{c}$ is the simply connected complex analytic Lie group with Lie algebra $g_{c}$ (here for any vector space $V$ defined over $R$ we denote its complexification by $V_{c}$; in particular $g_{c}$ is the complexification of $g$ ) then $G \subset G_{c}$. Fix a maximal compact subgroup $K$ of $G$. Assume further that $r k(G / K)=1$. This paper has two principal sections. In $\S I$ we characterize the invariant transforms of functions in $\mathscr{C}^{p}(G: F)(F \subset K,|F|<\infty)$; $\S$ II deals with the characterization of the orbital integrals of such functions.

Let $H$ be a $\theta$-stable Cartan subgroup of $G$ which is maximally split; in the case $r k(G)=r k(K)$ let $B$ in a Cartan subgroup of $G$ contained in $K$. It is known that $\hat{B} \subset \mathscr{L}_{B} \cong b_{c}^{*}$, and to each $A \in$ $\mathscr{L}_{B}^{\prime}$, the regular elements of $\mathscr{L}_{B}$, there corresponds $\omega(\Lambda) \in \widetilde{G}^{2}$ (we denote by $\widehat{G}^{p}$ the equivalence classes of those representations whose $K$-finite matrix coefficients are $\mathscr{L}^{p}$ functions on $G$ ). Fix $\pi_{1} \in \omega(\Lambda)$ for each $\Lambda \in \mathscr{L}_{B}^{\prime}$. If $H=H_{K} A\left(H_{K}=H \cap K, A\right.$ a vector group) $\chi \in \hat{H}_{K}^{\prime}$ (the prime denoting the regular elements of $\hat{H}_{K}$ ) $\nu \in a_{c}^{*}$ then we can define $\pi_{x, \nu}$, a principal series representation of $G$.

We denote by $\widetilde{G}$ (resp. $\hat{G}$ ) the set of all infinitesimal equivalence classes of irreducible admissible (resp. unitary) representations of $G$. If $\pi$ is an admissible representation we denote its global character by $\theta_{\pi}$. It is known that $\theta_{\pi}$ is a distribution which is given by a function which is (real) analytic on the regular set of $G$; we again denote this function by $\theta_{\pi}$. If $\pi, \nu$ are equivalent irreducible admissible representations then $\theta_{\pi}=\theta_{\nu}$. Hence to each $\omega \in \widetilde{G}$ there corresponds a character $\theta_{\omega}$; characters of the class $\omega=\omega(\Lambda)\left(\Lambda \in \mathscr{L}_{B}^{\prime}\right)$ will be denoted $\theta_{A}$ or $\theta_{\omega}$ and characters of the class $\left[\pi_{x, \nu}\right]$ will be denoted $\theta_{x, \nu}$. In $\S$ I we introduce the transform

$$
\hat{f}(\omega)=\int_{G} \theta_{\omega}(x) f\left(x^{-1}\right) d x=\left(\theta, f_{\omega}\right) \quad(\omega \in \widetilde{G}) .
$$

We refer to this as the invariant Fourier transform of $f$. Let $\hat{f}(\omega(\Lambda))=\hat{f}(\Lambda)$ and $\hat{f}\left(\chi_{: \nu}\right)=\hat{f}\left(\left[\pi_{\chi, \nu}\right]\right)$. Then we show that there exists a list of properties involving; (a) holomorphy and growth properties of $\hat{f}(\chi)$ as a function on a strip $\mathscr{F}_{0}(2 / p-1)$; (b) relations between $\hat{f}(\Lambda)$ and $\hat{f}\left(\chi_{:} \nu\right)$ which reflect the fact that for $\Lambda \in \mathscr{L}_{B}^{\prime}$ such that 
$\omega(\Lambda) \notin \widehat{G^{p}}$ then $\pi_{\Lambda}$ is embedded in $\pi_{\chi, \nu}$ (for suitable $\chi$ and $\nu \in \mathscr{F}_{c}(2 / p-1)$ ); which characterize the image of $\mathscr{C}^{p}(G: F)$ under the map $f \rightarrow \widehat{f}$. We denote this space of functions by $\mathscr{C}^{p}(C(G): F)$. The most difficult part of the proof that $\mathscr{C}^{p}(C(G): F)$ is the image of $\mathscr{C}^{p}(G: F)$ is the question of surjectivity. We shall now detail the major steps in the proof of surjectivity.

Let $L \in \mathscr{C}^{p}(C(G): F)$ and let us form the wave packets (here let $\left.L(\Lambda)=L(\omega(\Lambda)), L(\chi: \nu)=L\left(\left[\pi_{\chi, \nu}\right]\right)\right)$

$$
\begin{aligned}
\phi_{L}(x)= & \frac{1}{|W(G / B)|} \sum_{\Lambda \in \mathscr{S}_{B}^{\prime}} d(\Lambda) \operatorname{dim}\left(\mathscr{H}_{1, F}\right) L(\Lambda) \theta^{F}(x) \\
& +D(G / A) \sum_{\chi \in \hat{H}_{K}^{\prime}} d(\chi) \operatorname{dim}\left(\mathscr{H}_{\chi, F}\right) \int_{i a^{*}} L(\chi: \nu) \theta_{\chi, \nu}^{F}(x) \mu(\chi: \nu) d \nu
\end{aligned}
$$

where if $\omega \in \widetilde{G}, \theta_{\omega}^{F}$ denotes the sum of the $K$-Fourier coefficients of $\theta_{\omega}$ corresponding to $\delta \in F, d(\Lambda)$ equals the formal degree of $\pi_{\Lambda}$, $D(G / A)$ and $\mu(\chi: \nu)$ are defined in [8]. Then it is known (cf. [8]) that $\phi_{L} \in \mathscr{C}^{2}(G: F)$ and $\hat{\phi}_{L}(\chi: \nu)=L(\chi: \nu)\left(\chi \in \hat{H}_{K}^{\prime}, \nu \in i a^{*}\right), \hat{\phi}_{L}(\Lambda)=L(\Lambda)$ $\left(\Lambda \in \mathscr{L}_{B}^{\prime}\right)$. In fact more can be said. In the definition of $\phi_{L}$ let the sum over $\mathscr{L}_{B}^{\prime}$ (resp. the integral over $i a^{*}$ ) be denoted by $\dot{\phi}_{L}^{B}$ (resp. $\left.\phi_{L}^{H}\right)$. It can be shown that $\phi_{L}^{B}, \phi_{L}^{H} \in \mathscr{C}^{2}(G: F)$. Of course even if $L$ satisfies the requisite properties $\phi_{L}^{B}, \phi_{L}^{H}$ will not in general belong to $\mathscr{C}^{p}(G: F)$. This follows since if $S \subset \mathscr{L}_{B}^{\prime}, \phi_{L, S}=$ $\sum_{\Lambda \in S} d(\Lambda) \operatorname{dim}\left(\mathscr{L}_{\Lambda, F}\right) L(\Lambda) \theta_{\Lambda}^{F}, \mathscr{L}_{B, p}=\left\{\Lambda \in \mathscr{L}_{B}^{\prime}: \omega(\Lambda) \in \widehat{G}^{p}\right\}, \mathscr{L}_{B, p}^{\perp}=\left\{\Lambda \in \mathscr{L}_{B}^{\prime}:\right.$ $\left.\Lambda \notin \mathscr{L}_{B, p}\right\}, \phi_{L, p}=\phi_{L, \mathscr{L} B, p}, \phi_{L, p}^{\perp}=\phi_{L, \mathscr{L}} \frac{1}{B, p}$, then $\phi_{L}^{B}=\phi_{L, p}+\phi_{L, p}^{\perp}$ and $\phi_{L, p}$ is perpendicular to $\phi_{L, p}^{\perp}$ in the $L^{2}$-inner product. In fact we can write $\mathscr{\mathscr { C }}^{2}(G: F)=\dot{\mathscr{C}}^{p}(G: F)+\dot{\mathscr{C}}^{p}(G: F)^{\perp}$ (orthogonal direct sum). Hence if $\phi_{L}^{B}, \phi_{L}^{H} \in \mathscr{C}^{p}(G: F)$ then $\phi_{L, p}^{\perp} \in \mathscr{\mathscr { C }}^{p}(G: F) \cap \mathscr{\mathscr { C }}^{p}(G: F)^{\perp}=\{0\}$. One would hope then to be able to show that $\phi_{L}^{H}+\phi_{L, p}^{\perp} \in \mathscr{C}^{p}(G: F)$. This is not in general possible. Instead we proceed as follows.

We produce given $L$ an auxillary function $\beta_{L} \in C_{c}^{\infty}(G: F)$ such that $\left(\beta_{L}\right)^{\wedge}(\Lambda)=L(\Lambda)\left(\Lambda \in \mathscr{L}_{B, p}^{\perp}\right)$ and $\phi_{L-\left(\beta_{L}\right)}^{H} \in \mathscr{C}^{p}(G: F)$. Hence if we denote by $\phi_{L_{0}}^{B}$ the wave packet formed with the modified function $L_{0}$ where $L_{0}(\Lambda)=0$ if $\Lambda \in \mathscr{L}_{B, p}^{\perp}$, and $L_{0}(\Lambda)=L(\Lambda)\left(\Lambda \in \mathscr{L}_{B, p}\right)$ and set (here we use the notation of [8])

$$
f_{L}=\phi_{L-\left(\beta_{L}\right)}^{H} \hat{)}+\left(\beta_{L}\right)_{A}+\phi_{\left(\beta_{L}\right) \wedge, p}^{\perp}+\phi_{L_{0}}^{B}
$$

then we have by [8] that

$$
\begin{aligned}
\hat{f}_{L}\left(\chi_{: \nu)}\right. & =\left(\phi_{L-\left(\beta_{L}\right) \wedge}^{H}\right)^{\wedge}\left(\chi_{:} \nu\right)+\left(\left(\beta_{L}\right)_{A}\right)^{\wedge}\left(\chi_{: \nu}\right) \\
& =L\left(\chi_{: \nu}-\left(\beta_{L}\right)^{\wedge}\left(\chi_{:} \nu\right)+\left(\beta_{L}\right)^{\wedge}\left(\chi_{: \nu)}\right.\right. \\
& =L\left(\chi_{: \nu}\right) .
\end{aligned}
$$

By the orthogonatily relations for the discrete series characters we 
have for $\Lambda \in \mathscr{L}_{B, p}^{1}$

$$
\left(f_{L}\right)^{\wedge}(\Lambda)=\left(\phi^{\perp}{ }_{\left(\beta_{L}\right), \hat{p}}\right)^{\wedge}(\Lambda)=\left(\beta_{L}\right)^{\wedge}(\Lambda)=L(\Lambda)
$$

and for $\Lambda \in \mathscr{L}_{B, p}$ we have

$$
\left(f_{L}\right)^{\wedge}(\Lambda)=\left(\phi_{L_{0}}^{B}\right)^{\wedge}(\Lambda)=L_{0}(\Lambda)=L(\Lambda) .
$$

In $\S$ II we introduce the invariant orbital integrals, $F_{f}$; we denote the restriction of this function to the regular points of $B$ (resp. $H$ ) by $F_{f}^{B}$ (resp. $F_{f}^{H}$ ). The functions $F_{f}^{B}, F_{f}^{H}$ are $L^{1}$ on $B$ and $H$ respectively. Hence we can take their Fourier transforms. It is known (cf. [19]) that $\hat{F}_{f}^{H}$ has a simple relation with $\hat{f}_{H}$ and $\hat{F}_{f}^{B}(\Lambda)$ a complicated relation with $\hat{f}_{B}$. Nevertheless we are able to transcribe our conditions defining $\mathscr{C}^{p}(C(G): F)$ over onto $F_{f}^{B}$ and $F_{f}^{H}$ which then allows us to characterize these functions.

One remark is in order which is explicated in more detail in $\S$ II. The Fourier transforms of $F_{f}^{B}$ and $F_{f}^{H}$ are defined for all $\Lambda \in \mathscr{L}_{B}$ and $\chi \in \hat{H}_{k}$ (not just the regular elements). We were then forced to extend our definition of the invariant transform to include the singular elements of $\Lambda \in \mathscr{L}_{B}$ and $\chi \in \hat{H}_{k}$.

The importance of these characterizations is, apart from their natural place in the harmonic analysis of $G$, that they occur in the study of the Selberg trace formula. In fact if $\Gamma$ is a discrete compact subgroup of $G, L$ denotes the left regular representation of $G$ on $L^{2}(G / \Gamma)$ then it is known that

$$
L=\sum_{\omega \in \hat{G}} m_{\omega} \omega
$$

(i.e., $L$ is discretely decomposable into a direct sum of irreducible unitary representations with finite multiplicities). A natural problem is to determine the integers $m_{\omega}$.

For $f \in \mathscr{C}^{1}(G)$ the operator $L(f)$ is of trace class and we have

$$
\operatorname{tr} L(f)=\sum_{\omega \in G} m_{\omega} \hat{f}(\omega) .
$$

On the other hand, we can write

$$
\operatorname{tr} L(f)=\sum_{\{b \mid} \mu\left(G_{y} / \Gamma_{h}\right) \int_{G / G_{y}} f\left(y^{x}\right) d G / G_{y}(x)
$$

where $\{y\}$ runs through the conjugacy classes in $\Gamma, G_{y}=\operatorname{Cent}_{G}(y)$, $\Gamma_{y}=\Gamma \cap G_{y}$, and $\mu\left(G_{y} / \Gamma_{y}\right)$ is the volume of $G_{y} / \Gamma_{y}$.

In order to obtain information about the $m_{\omega}$ (for instance obtaining limit formulas (cf [4])) one can attempt to express the right hand side of the last equation above in terms of invariant transforms for functions $f$ on $G$ whose Fourier transform can be 
explicitly computed; the easiest way to do this is to start on the Fourier transform side and inverse transform back to the group. This of course requires the above characterizations.

For other papers on this subject see also [19].

Notation. We retain the notation of the introduction and all other notation not explained below is as in [15].

Let $M$ be a differentiable manifold, $W$ (resp. $(W, \gamma))$ be a finite dimensional vector space (resp. a finite dimensional double unitary $K$-module). The space of infinitely differentiable functions on $M$ taking values in $W$ and those of compact support (resp. the $\gamma$ spherical infinitely differentiable functions and those of compact support) will be denoted by $C(M: W)$ and $C_{c}^{\infty}(M: W)\left(\right.$ resp. $C^{\infty}(M: W: \gamma)$, $C_{c}^{\infty}(M: W: \gamma)$ ) when $W=C$ we suppress the $W$ in the notations $C^{\infty}(M: W)$ and $C_{c}^{\infty}(M: W)$.

If $A$ is an arbitrary set $B \subseteq A$ we denote by [B] the compliment of $B$ in $A$. Further if $B$ is a finite subset of $A$ then we denote the number of elements in $B$ by the notation $|B|$.

If $V$ is a vector space over $\boldsymbol{R}$ we shall denote by $V_{c}$ its complexification; i.e., $V_{c}=V \otimes{ }_{R} C$. Let $V^{*}$ (resp. $\left.V_{c}^{*}\right)$ denote the real (resp. complex) dual of $V$ (resp. $V_{c}$ ).

For an arbitrary Lie group $L$ let us denote by $\hat{L}$ the set of equivalence classes of irreducible unitary representations of $L$.

Suppose now that $M$ is as above and there exists a topological action of $K$ on $M$ both on the right and left. If $\xi \in \hat{K}$ let $\chi_{\xi}$ denote the character of $\xi$ and $\rho_{\iota}, \rho_{r}$ denote the left and right actions of $K$ on $M$. We shall write for $F \subset \hat{K},|F|<\infty, C^{\infty}(M: F)$ and $C_{c}^{\infty}(M: F)$ for the subspaces of $C^{\infty}(M)$ and $C_{c}^{\infty}(M)$ respectively of those functions $f$ which satisfy the following:

$$
d(\xi) \int_{K} \operatorname{conj} \chi_{\xi}(k) f\left(\rho_{\iota}(k) m\right) d k=d(\xi) \int_{K} f\left(m \rho_{r}(k)\right) \operatorname{conj} \chi_{\xi}(k) d k=f(m)
$$

where $d(\xi)$ denotes the degree of $\xi$. If $A \subset C^{\infty}(M)$ then we shall write $A(F)$ for the corresponding subspace of $C^{\infty}(M: F)$.

Let $\mathfrak{g}=\mathfrak{l}+\mathfrak{g}$ be a Cartan decomposition of $\mathfrak{g}, \theta$ the corresponding Cartan involution of $g$ (we also use $\theta$ for the involution of $G$ ). Let $\mathfrak{h}$ be a $\theta$ stable Cartan subalgebra of $\mathfrak{g}$ with maximal vector part. Put $\mathfrak{a}=\mathfrak{h} \cap \mathfrak{s}$ and assume $\operatorname{dim} \mathfrak{a}=1$. If $\operatorname{rank}(G)=\operatorname{rank}(K)$ let $B$ be a Cartan subgroup $G$ contained in $K$.

For any Cartan subalgebra $\mathfrak{l} \subset \mathfrak{g}$ let $\Delta\left(\mathfrak{g}_{c}, \mathfrak{l}_{c}\right)$ denote the nonzero roots of the pair $\left(\mathfrak{g}_{c}, \mathfrak{l}_{c}\right)$. We denote by $W\left(\mathfrak{g}_{c}, \mathfrak{l}_{c}\right)$ the group generated by the reflections $s_{\alpha}\left(\alpha \in \Delta\left(\mathfrak{g}_{c}, \mathfrak{l}_{c}\right)\right)$. When $\mathfrak{l}$ is understood we shall simply write $W$; we refer to $W$ as the Weyl group of the pair $\left(\mathfrak{g}_{c}, \mathfrak{l}_{c}\right)$. 
If we have an action of $A$ on $B$ we denote by $B^{A}$ the set of $A$-invariants in $B$.

Let $M=\operatorname{Cent}_{K}(\mathfrak{a}), M^{\prime}=\operatorname{Norm}_{K}(\mathfrak{a})$. The $M$ and $M^{\prime}$ are compact groups, and $W(A)=M^{\prime} / M$ is a finite group. If $\chi \in \hat{M},(V, \sigma)$ is an $M$-module in the class $\chi, \nu \in \mathfrak{a}_{c}^{*}$ and $w \in M^{\prime}$ then we shall write $w \sigma$ and $w \nu$ for the following

$$
\begin{aligned}
& w \sigma(m)=\sigma\left(w^{-1} m w\right) \quad(m \in M) \\
& w \nu(H)=\nu\left(w^{-1} H w\right) \quad(H \in \mathfrak{a}) \text {. }
\end{aligned}
$$

Obviously if $\sigma^{\prime}$ is also of class $\chi$ then $w \sigma^{\prime}$ and $w \sigma$ are again of the same class, i.e., $M^{\prime}$ acts on $\hat{M}$. Note also that the action of $M^{\prime}$ on $\sigma$ and $\nu$ depends only on the residue class of $w$ in $W(A)$. If $s \in W(A)$ we shall have occasion to write $s \sigma ; s \chi$ and $s \nu$ which then have their obvious meaning.

Let $P(A)$ denote the set of all parabolic subgroups of $G$ whose split component is $A$. Fix $Q \in P(A)$, and let $Q=M A N_{Q}$ be its Langlands decomposition (note that $M$ is as above). We put $\mathfrak{m}_{1}=$ $\mathfrak{m}+\mathfrak{a}$ and note that $\mathfrak{h} \subset \mathfrak{m}_{1}$, and is a Cartan subalgebra of the reductive algebra $m_{1}$. Let $W=W\left(\mathfrak{g}_{c}, \mathfrak{h}_{c}\right), W_{1}=W\left(\mathfrak{m}_{1 c}, \mathfrak{h}_{c}\right)$. Then in a natural way we may consider $W_{1}$ as a subgroup of $W$.

To each $\theta$-stable Cartan subgroup of $G$ we shall associate a series of representations as follows. Let $Q$ be as above, $Q=M A N$. Let $\chi \in \hat{M}, \quad \sigma \in \chi, \nu \in a_{c}^{*}$ and put $\pi_{\chi, \nu}=\pi_{Q, \chi, \nu}=\operatorname{Ind}_{Q}^{G}\left(\sigma \otimes \xi_{\nu}\right)$ where $\xi_{\nu}(a)=e^{\nu(\log a)}$ (as the exponential map restricted to $\mathfrak{g}$ is a diffeomorphism then if $x=\exp X$ we write $X=\log x$ ), and $\sigma \otimes \xi_{\nu}$ is extended to $Q$ by making it trivial on $N$. We shall assume that $\pi_{x, \nu}$ acts by right translation and it represents $G$ on $\mathscr{H}_{x}=\mathscr{H}_{Q, a}$, in the compact picture (i.e., functions defined on $K$ ), or on $\mathscr{H}_{x, \nu}=\mathscr{H}_{Q, x, \nu}$, in the noncompact picture (i.e., functions defined on $G$ )).

Let $\pi_{Q}(\nu)=\langle\nu, \alpha\rangle\left(\nu \in \mathfrak{a}_{c}^{*}\right)$ where $\alpha$ denotes the unique simple root of $\Delta(\mathfrak{g}, \mathfrak{a}),\langle\cdot, \cdot\rangle$ denotes the killing form on $a_{c}^{*}$. Let $\mathscr{F}=(-1)^{1 / 2} a^{*}$, $\mathscr{F}_{c}=a_{c}^{*}$ and $\mathscr{F}_{r}=a^{*}$. We shall say that $\nu \in \mathscr{F}_{c}$ is regular if $\pi_{Q}(\nu) \neq 0$. We denote the set of regular elements of $\mathscr{F}_{,} \mathscr{F}_{c}$, and $\mathscr{F}_{k}$ by $\mathscr{F}^{\prime}, \mathscr{F}_{c}^{\prime}$, and $\mathscr{F}_{r}^{\prime}$ respectively.

It is known (cf. [11], Lemma 13.3) that $\pi_{x, \nu}$ is irreducible for all $\nu \in \mathscr{F}^{\prime}$. Moreover $\pi_{\chi, \nu}$ is unitarily equivalent to $\pi_{s \chi, s \nu}$ for all $\nu \in \mathscr{F}^{\prime}, s \in W(A)$. Hence there exists an isometry $\mathscr{A}_{Q \mid Q}: \mathscr{H}_{\chi, \nu} \rightarrow$ $\mathscr{H}_{s x, s \nu}$ such that

$$
\mathscr{A}_{Q \mid Q}(s: \chi: \nu) \pi_{\chi, \nu}(x)=\pi_{s \chi, s \nu}(x) \cdot \mathscr{A}_{Q \mid Q}(s: \chi: \nu) \quad(x \in G) \text {. }
$$

Moreover, for $Q, s$, and $\chi$ fixed, the function $\nu \rightarrow \mathscr{A}_{Q \mid Q}(s: \chi: \nu)$ has a meromorphic extension to $\mathscr{F}_{c}$.

Let $\theta_{\chi, \nu}$ denote the global character of $\pi_{\chi, \nu}$. It follows from 
the above that $\theta_{s \chi, s \nu}=\theta_{\chi, \nu}$ for all $\chi \in \hat{M}, \nu \in \mathscr{F}^{\prime}, s \in W(A)$.

Suppose that $r k(G)=r k(K)$ and $B$ is as before a Cartan subgroup of $G$ contained in $K$. Then there exists a lattice $\mathscr{L}_{B} \subset \underline{b}_{c}^{*}$ such that $\mathscr{L}_{B}$ is isomorphic to $\hat{B}$. Let $W(G / B)$ denote the finite group $\operatorname{Norm}_{G}(B) / B$. Then $W(G / B)$ acts on $\mathscr{L}_{B}^{\prime}$ (the regular elements of $\mathscr{L}_{B}$ ). Let $\mathscr{L}_{B}^{+}$be a fundamental domain for this action. To each $\Lambda \in \mathscr{L}_{B}$ Harish-Chandra has associated a representation $\omega(\Lambda)$ whose matrix elements are $L^{2}$ functions on $G$ (hereafter let us write $\widehat{G}^{2}$ and more generally $\widehat{G}^{p}$ for the equivalence classes of irreducible unitary representations of $G$ whose $K$-finite matrix coefficients are $L^{p}$ summable on $G$ ). It is known that if $\Lambda_{1}, \Lambda_{2} \in \mathscr{L}_{B}$ then $\omega\left(\Lambda_{1}\right)$ is equivalent to $\omega\left(\Lambda_{2}\right)$ if and only if $\Lambda_{1}=s \Lambda_{2}$ for some $s \in$ $W(G / B)$. In particular, $\mathscr{L}_{B}^{+}$uniquely parameterizes the class of representations corresponding to $B$. We shall denote by $\mathscr{H}_{A}$ the representation space of $\omega(\Lambda)$.

Let us now fix $F \subset \hat{K},|F|<\infty$. If $L$ is a Lie group, $N$ a compact subgroup of $L, \pi$ a representation of $L$ which when restricted to $N$ is unitary, then we shall write for $\delta \in \hat{N},[\pi: \delta]_{N}$ for the multiplicity of $\delta$ in the direct sum decomposition of $\pi$ restricted to $N$. With this notation let us put

$$
\begin{aligned}
& \widehat{M}(F)=\left\{\chi \in \widehat{M}:[\delta: \chi]_{M} \geqq 1 \text { for some } \delta \in F\right\}, \\
& \widehat{G}^{p}(F)=\left\{\omega \in \widehat{G}^{p}:[\omega: \delta]_{K} \geqq 1 \text { for some } \delta \in F\right\}
\end{aligned}
$$

then $|\hat{M}(F)|<\infty$, and $\left|\hat{G}^{p}(F)\right|<\infty$, and we have by the Frobenius reciprocity theorem that $\left[\pi_{x, \nu}: \delta\right] \neq 0$ for some $\delta \in F$ if and only if $\chi \in \hat{M}(F)$.

Let $\pi$ be a representation of $G$ on a Hilbert space $\mathscr{H}$. If $\delta \epsilon$ $\hat{K}$ let us write $\mathscr{H}_{\delta}$ for the isotypic component of $\mathscr{H}$ corresponding to $\delta$. Further, if $F \subset \hat{K}$ and $|F|<\infty$ let us put

$$
\mathscr{H}_{F}=\sum_{\delta \in F} \mathscr{H}_{\delta} \text {. }
$$

Let

$$
d(m)=d_{Q}(m)=\left(\operatorname{det} \operatorname{Ad}_{m \underline{n}_{Q} Q}\right)^{1 / 2} \quad(m \in M A)
$$

and

$$
\rho(H)=\rho_{Q}(H)=\frac{1}{2} \operatorname{tr}\left(\operatorname{ad}_{H \mid \underline{Q} Q}\right) \quad(H \in \underline{a}) .
$$

Let

$$
A^{+}=A^{+}(Q)=\left\{a \in A: e^{\alpha(\log a)}>1\right\}
$$

where $\alpha=\alpha_{Q}$ is the unique simple root in $\Delta(\mathfrak{g}, \mathfrak{a})$.

We shall denote the enveloping algebra of $\mathfrak{g}_{c}$ by $\mathfrak{B}$; if $\mathfrak{l}$ is a 
subalgebra of $\mathfrak{g}$ we shall denote the subalgebra of $B$ generated by $\mathfrak{l}_{c}$ and 1 by $\mathfrak{l}$. The symmetric algebra of $\mathfrak{g}_{c}$ will be denoted by $\mathscr{S}\left(\mathfrak{g}_{c}\right)$; elements of $\mathscr{S}\left(\mathfrak{g}_{\mathrm{c}}\right)$ will be treated as directional derivatives of function on $\mathfrak{g}_{c}$.

I.1. Some properties of representations. Let $0<p \leqq 2, a \in \mathbb{B}$ and $r \in \boldsymbol{R}$. For $f \in C^{\infty}(G)$ let

Put

$$
\nu_{a, r}^{p}(f)=\sup _{G} \Xi^{-2 / p}(1+\sigma)^{r}|a f| .
$$

$$
\mathscr{C}^{p}(G)=\left\{f \in C^{\infty}(G): \nu_{a, r}^{p}(f)<\infty \text { for all } a \in \mathbb{S}, r \in \boldsymbol{R}\right\} \text {. }
$$

Note that we use only one sided derivatives but we shall now restrict to a $K$-finite subspace of $\mathscr{C}^{p}(G)$ on which the two-sidedderivative seminorms and the one sided induce the same topology.

Fix $F \cong \hat{K},|F|<\infty$. For $\delta \in F$ let $\chi_{\dot{\delta}}$ denote the character of $\delta$ and set $\xi_{\dot{o}}=d(\delta) \operatorname{conj} \chi_{\dot{\delta}}$. Set

$$
\mathscr{C}^{p}(G: F)=\left\{f \in \mathscr{C}^{p}(G): \int_{K} \xi_{F}(k) f\left(k^{-1} x\right) d k=\int_{k} \xi_{F}\left(k^{-1}\right) f(x k) d k=f(x)\right\} .
$$

The following is material which we will need in order to form the auxillary function mentioned in the introduction. The following results can be found in [10]; all unexplained notations are as in that paper.

Let $\pi$ be an admissible representation of finite length. Set $\mathscr{F}_{c}^{-}=\left\{\mu=\nu+i \gamma \in \mathscr{F}_{c}: \nu(H) \leqq 0\right.$ for all $\left.H \in \mathfrak{a}^{-}(Q)\right\}$ where if $\alpha$ is the unique simple root of the pair $\Delta(\mathfrak{a}, \mathfrak{a})$ then $\mathfrak{a}^{-}(A)=\{H \in \mathfrak{a}: \alpha(H)<0\}$. Also set

$$
I_{Q}(\pi)=\left\{\nu \in \mathscr{F}_{c}: \operatorname{Hom}_{(g, K)}\left(\pi: \pi_{Q, \chi, \nu}\right) \neq\{0\} \text { for some } \chi \in \hat{M}\right\} .
$$

We denote by $\mathscr{E}_{Q}^{0}(\pi)$ the set of leading exponents of $\pi$ along $Q$.

LEMMA 1. (Milicic). Let $\pi$ be an admissible representation of finite length. Then the set $\mathscr{E}_{Q}^{0}(\pi)$ equals the set of minimal exponents in $I_{Q}(\pi)$.

REMARK. The ordering in Lemma 2 is as follows. Let $L$ be the lattice generated by $\Delta(\mathfrak{q}, \mathfrak{a}), L^{+}$the cone of sums of positive roots. We write $\lambda \gg \mu\left(\lambda, \mu \in \mathscr{F}_{0}\right)$ if $\lambda-\mu \in L^{+}$.

Suppose that $r k(G)=r k(K), \mathfrak{b}=L A(B)$. Put $\Phi=\Delta(\mathfrak{g}, \mathfrak{b})$ and for $\alpha \in \Phi$ set

$$
k(\alpha)=1 / 4 \sum_{\beta \in \Phi}|(\alpha, \beta)|
$$

Lemma 2. (Milicic [10]). Let $\kappa>0$. Then for every $\Lambda \in L_{B}^{+}$ 
the following are equivalent:

(1) $|(\Lambda, \alpha)| \geqq \kappa k(\alpha)$ for all noncompact $\alpha \in \Phi$

(2) every leading exponent of $\pi_{A}$ along $Q$ lies in $\kappa \rho_{Q}+\mathscr{F}^{-}$.

Lemma 3. ([10], [17]) (i) A necessary and sufficient condition that $\pi_{1} \in \hat{G}^{p}$ is that $|(\Lambda, \alpha)|>(2 / p-1) k(\alpha)$ for all noncompact $\alpha$.

(ii) If $\pi_{1} \in \widehat{G}^{p}$ and $c(x)$ is a $K$-finite matrix coefficient of $\pi_{A}$ then there exists a constant $D>0$ such that

$$
|c(x)| \leqq D \Xi^{2 / p+\varepsilon_{0}}(x)
$$

for some $\varepsilon_{0}>0$.

Lemma 4. Let $\omega \in \widehat{G}^{2}$ and $0<p<2$. Then

$$
I_{Q}\left(\pi_{\omega}\right) \cap \mathscr{F}_{c}\left(\frac{2}{p}-1\right) \neq \phi
$$

if and only if $w \in \widehat{G}^{2} / \widehat{G}^{p}$.

Proof. It is a surprising fact (although in the rank one case it is more or less obvious) that one can deduce global estimates from leading exponents. That is, if for all $\lambda \in \mathscr{E}_{Q}^{0}(\pi), \operatorname{Re} \lambda-\gamma \rho_{Q} \leqq$ 0 (on $\left.\mathfrak{a}^{-}(Q)\right) \gamma>0$, then for any $K$-finite matrix coefficient $c$, there exists a constant $D>0$ such that $|c(x)| \leqq D \Xi^{r}(x)$. Hence from Lemmas 2 and 3 above it is easy to deduce that $\mathscr{E}_{Q}^{0}\left(\pi_{\omega}\right) \cap \mathscr{F}_{c}(2 / p-1) \neq$ $\phi$ if and only if $\omega \in \widehat{G}^{2} / \widehat{G}^{p}$. The lemma then follows from Lemma 1 above.

From the estimates of [15] it is easy to deduce that if $\chi \in \hat{M}$, $\nu \in \mathscr{F}_{c}(2 / p-1)$ then $\alpha \rightarrow \theta_{\chi, \nu}(\alpha)$ is a continuous linear functional on $\mathscr{C}^{p}(G)$. The following lemma is also a simple consequence of these estimates.

Lemma 5. Let $\chi \in M, \nu \in \mathscr{F}_{c}(2 / p-1)$. If $\theta_{\chi, \nu}=\theta_{1}+\cdots+\theta_{\iota}$ where each $\theta_{i}$ is an irreducible character then $\alpha \rightarrow \theta_{i}(\alpha)$ is a continuous linear functional on $\mathscr{C}^{p}(G)$.

If $\chi \in \hat{M}, \nu \in \mathscr{F}_{c}$, and $G(\chi: \nu: x)=\theta_{\chi, \nu}^{F}(x)$ then for each $\chi \in \hat{M}$, $G(x)$ is analytic on $\mathscr{F}_{c} \times G$. Let us denote by $\theta_{\chi, \nu, k}^{F}$ the distribution given by the function $G\left(\chi: \nu ; \partial^{k}(\nu): x\right)$.

Fix $0<p<2$. Let $U_{p}$ denote the union of the sets (1), (2), and (3) specified in $\S 7$ of [15] intersected with $\mathscr{F}_{c}(2 / p-1)$. Set $V_{p}=U_{p} \cup\left(\cup I_{Q}(\omega) \cap \mathscr{F}_{c}(2 / p-1)\right)$. For every $\chi \in \hat{M}(F), \quad t \in W(A)$, $\zeta \in V_{p} \omega \in \widehat{G}^{2}(F) \backslash \widehat{G}^{p}(F)$ we can write $\theta_{\alpha, t \zeta}^{F}$ as a sum of irreducible characters. Let $\mathscr{E}_{p}$ denote the set of $F$-Fourier components of all 
these characters union with the distributions $\theta_{\alpha, t \xi, k}^{F}(\chi, t, \zeta$ as above and $0 \leqq k \leqq 0_{t}(\zeta)-1$ (cf. [15])). Recall that $\left.\theta_{\omega} \in \mathscr{E}_{p}\left(\omega \in \hat{G}^{2}(F)\right) \backslash \hat{G}^{p}(F)\right)$.

Let $\mathscr{B}_{p}$ be a basis for the linear space spanned by $\mathscr{E}_{p}$ chosen as follows. To the characters $\theta_{\omega}\left(\omega \in \widehat{G}^{2}(F) \mid \hat{G}^{p}(F)\right)$ adjoint linearly independent elements from the characters in the set $\mathscr{E}_{p}$. Next adjoin to this set linearly independent elements from the set $\left\{\theta_{\chi, t \zeta ; k}: \chi \in \widehat{M}(F), t \in W(A), \zeta \in V_{p}, 1 \leqq k \leqq 0_{t}(\zeta)-1\right\}$. For $\chi, t, \zeta, k$ as usual let us define constants $C_{p}\left(\theta_{\chi, t \zeta, k}^{F}: \theta\right)\left(\theta \in \mathscr{B}_{p}\right)$ by the equation

$$
\theta_{\chi, t \zeta, k}^{F}=\sum_{\theta \in \mathscr{P}_{p}} C_{p}\left(\theta_{\chi, t \zeta, k}^{F}: \theta\right) \theta
$$

Further for any $\theta^{\prime} \in \mathscr{E}_{p}$ let $C_{p}\left(\theta^{\prime}: \theta\right)$ be defined by

$$
\theta^{\prime}=\sum_{\theta \in \mathscr{Q}_{p}} C_{p}\left(\theta^{\prime}: \theta\right) \theta
$$

For $0<p_{j}<2, p_{j}$ sufficiently small $(j=1,2) \quad U_{p_{1}}=U_{p_{2}}$ and $V_{p_{j}} \supset \bigcup_{\omega \in \hat{G}^{2}(F) \mid \hat{G}_{(F)}\left(I_{Q}\right.} I_{Q}(\omega)$. Let us fix such a $p$ and replace the notations $V_{p}, \mathscr{E}_{p}, \mathscr{B}_{p}$, and $C_{p}$ by $V, \mathscr{E}, \mathscr{B}$, and $C$.

Fix an open neighborhood $V_{0}$ of $1 \in G$ with compact closure. By their linear independence and analyticity on the regular elements of $G$, we can choose for each $\theta \in \mathscr{B}_{p}, \alpha_{\theta} \in C_{c}^{\infty}(G: F)$ such that $\operatorname{supp} \alpha_{0} \subseteq V_{0}$ and if

$$
\left(\alpha_{\theta}, \theta^{\prime}\right)=\int_{G} \alpha_{\theta}\left(x^{-1}\right) \theta^{\prime}(x) d x,
$$

then $\left(\alpha_{\theta}, \theta^{\prime}\right)=0$ if $\theta^{\prime} \in \mathscr{B}_{p}, \theta^{\prime} \neq \theta$, and $\left(\alpha_{\theta}, \theta\right)=1$. Further let us require that $\left(\alpha_{\theta}, \theta_{\omega}\right)=0\left(\omega \in G^{p}\right)$. This last condition is permissible by Lemma 4 . The functions $\alpha_{\theta}$ are by no means uniquely determined. However, we do have the following result. Define $\left(\alpha, \theta_{, \nu, k}^{F}\right)$ as in (3).

Proposition 1. With the above notation,

$$
\left(\alpha_{\theta}, \theta_{\chi, t \zeta, k}^{F}\right)=C\left(\theta_{\chi, t \zeta, k}^{F}: \theta\right) \text {. }
$$

More generally for any $\alpha \in C_{c}(G: F)$,

$$
\left(\alpha, \theta_{\chi, t \zeta, k}^{F}\right)=\sum_{\theta \in \mathscr{Q}} C\left(\theta_{\chi, t \zeta, k}^{F}: \theta\right)(\alpha, \theta) .
$$

Proof. (1) is obvious from (1) above. (2) follows from the bilinearity of the symbol $(\cdot, \cdot)$.

I.2. Definition and properties of the invariant transform. Let $C(G)$ denote the space of characters of quasi-simple admissible representations of $G$ which are of finite length. For $\theta \in C(G)$ and $\alpha \in C_{c}^{\infty}(G: F)$ let us write 


$$
\hat{\alpha}(\theta)=(\alpha, \theta)=\int_{\theta^{\prime}} \alpha\left(x^{-1}\right) \theta(x) d x .
$$

Here we use the fact that such characters are given by (analytic) functions on the regular set $G^{\prime}$ of $G$. For $\theta=\theta_{\chi, \nu}, \chi \in \hat{M}, \nu \in \mathscr{F}_{\circ}$ we shall sometimes write $\hat{\alpha}\left(\chi_{:} \nu\right)$ in place of $\hat{\alpha}\left(\theta_{\chi, \nu}\right)$ and for $\omega \in \hat{G}^{2}$ we shall frequently write $\hat{\alpha}(\omega)$ in place of $\hat{\alpha}\left(\theta_{\omega}\right)$. We shall refer to $\hat{\alpha}$ as the invariant Fourier transform of $\alpha$.

If $G$ is a complex valued function with domain $D \cong \hat{M} \times \mathscr{F}$. such that $\hat{M}(F) \times \mathscr{F} \subset D$ then set

$$
\phi_{G}(\chi: x)=\int_{\mathscr{F}} \theta_{\tilde{x}, \nu}^{F}(x) G(\chi: \nu) \mu(\chi: \nu) d \nu \quad(x \in G)
$$

and

$$
\left.\phi_{G}(x)=D(G / A) \sum d(\chi) d_{F}(\chi)^{-1} \phi_{G}(\chi: x) \quad \text { (sum over } \chi \in \hat{M}\right),
$$

where $d_{F}(\chi)=\operatorname{dim} \mathscr{H}_{\chi, F}$.

Let $\chi \in \hat{M}(F)$ and denote by $E_{F}(\chi)$ the orthogonal projection of $\mathscr{H}_{\chi}$ onto $\mathscr{H}_{x, F}$.

Lemma 1. Let $\alpha \in C_{c}^{\infty}(G: F)$. Then $\hat{\alpha}\left(\chi_{:} \cdot\right)$ is an entire function on $\mathscr{F}_{\mathrm{c}}$ of exponential type. Further,

(1) $\hat{\alpha}\left(s \chi_{:} s \nu\right)=\hat{\alpha}\left(\chi_{:} \nu\right)\left(s \in W(A), \chi \in \hat{M}, \nu \in \mathscr{F}_{c}\right)$

(2) $\hat{\alpha}\left(\chi_{:}: \nu\right) \equiv 0$ if $\chi \notin \hat{M}(F)$.

(3) $\hat{\alpha}(\omega)=0$ if $\omega \notin \hat{G}^{2}(F)$

(4) defining $\dot{\phi}_{\alpha}^{\hat{\alpha}}$ as in (2) above we have

$$
\hat{\phi}_{\alpha}\left(\chi_{:} \nu\right)=\hat{\alpha}(\chi: \nu) \quad\left(\chi \in \hat{M}(F), \nu \in \mathscr{F}_{c}\right) .
$$

Proof. The holomorphy and growth properties of $\hat{\alpha}(\chi: \nu)$ (as a function on $\mathscr{F}_{c}$ ) follow easily from the corresponding properties of $\mathscr{F}_{H}(\alpha)$ (cf. [15]).

(1) follows from the identity $\theta_{\chi, \nu}=\theta_{s x, s \nu}$. Statements (2) and (3) are obvious. To prove (4) let us call the left side of $(3) g\left(\chi_{:} \nu\right)$. Then

$$
g\left(\chi_{0}: \nu_{0}\right)=D(G / A) \sum_{\chi \in \hat{M}(F)} d(\chi) d_{F}(\chi)^{-1}\left(\phi_{\hat{\alpha}}, \theta_{\chi_{0}, \nu_{0}}\right) .
$$

By Theorem 2.1 of [8] we have (with $W\left(\chi_{0}\right)=\left\{s \in W(A): s \chi_{0}=\chi_{0}\right\}$ )

$$
g\left(\chi_{0}: \nu_{0}\right)=D(G / A) \sum_{s \in W / W\left(\chi_{0}\right)} d\left(s \chi_{0}\right) d_{F}\left(s \chi_{0}\right)^{-1}\left(\phi_{\hat{\alpha}}\left(s \chi_{0}\right), \theta_{\chi_{0}, \nu_{0}}\right)
$$

where the sum over $W / W\left(\chi_{0}\right)$ means over a complete set of representatives. Again applying Theorem 22.1 of [8] we have 


$$
\begin{gathered}
g\left(\chi_{0}: \nu_{0}\right)=D(G / A) \sum_{s \in W ; W\left(\chi_{0}\right)} d\left(s \chi_{0}\right) d_{F}\left(s \chi_{0}\right)^{-1} r c^{2} d\left(s \chi_{0}\right)^{-1} F_{0} \psi_{E_{F}\left(s \chi_{0}\right)}(1) \\
\quad \times \sum_{t \in W\left(s \chi_{0}\right)} \hat{\alpha}\left(s \chi_{0}: t s \nu_{0}\right) .
\end{gathered}
$$

By (3) of Lemma 9.1 of [8] we have the above

$$
\begin{aligned}
& =|W|^{-1} \sum_{s \in W / W\left(\chi_{0}\right)} \sum_{t \in W \mid W\left(s \chi_{0}\right)} \hat{\alpha}\left(s \chi_{0}: t s \nu_{0}\right) \\
& =|W|^{-1} \sum_{s \in W / W\left(\chi_{0}\right)} \hat{\alpha}\left(s \chi_{0}: s \nu_{0}\right)\left|W\left(s \chi_{0}\right)\right| \\
& =|W|^{-1} \mid W\left(\chi_{0}\right) \cdot\left[W: W\left(\chi_{0}\right)\right] \hat{\alpha}\left(\chi_{0}: \nu_{0}\right) .
\end{aligned}
$$

The last lines of equalities follow from (1) above and the obvious fact that $W\left(s \chi_{0}\right)=s W\left(\chi_{0}\right) s^{-1}$.

In the preceding section we defined the sets $\mathscr{E}$ and $\mathscr{B}$ and the constants $C\left(\theta_{x, t \tau, k}: \theta\right)$. For any scalar valued function $L$ defined on the space $C(G)$ such that for each $\chi \in \widehat{M}, \nu \rightarrow L(\chi: \nu)$ is $C^{\infty}$ let us put

$$
\beta_{L}(x)=\sum_{\theta \in \mathscr{O}} L(\theta) \alpha_{\theta}(x) \quad(x \in G)
$$

where if $\theta=\theta_{\chi, t \tau, k}$ with $k>0$ then by $L(\theta)$ we intend $L\left(\chi: t \zeta ; \partial^{k}(\nu)\right)$. As $|\mathscr{B}|<\infty$ then $\beta_{L} \in C_{c}^{\infty}(G: F)$.

Proposition 1. Let $\alpha \in C_{c}^{\infty}(G: F)$. Then,

(1) $\widehat{\beta}_{\hat{\alpha}}\left(\chi: t \zeta ; \partial^{k}(\nu)\right)=\widehat{\alpha}\left(\chi: t \zeta ; \partial^{k}(\nu)\right)$

for all $\chi \in \widehat{M}(F), t \in W(A), \zeta \in V, 0 \leqq k \leqq 0_{t}(\zeta)-1$.

(2) $\hat{\beta}_{\hat{\alpha}}(\omega)=\hat{\alpha}(\omega)\left(\omega \in \hat{G}^{2}\right)$.

Proof. By Proposition I.1.1 we have

$$
\begin{aligned}
\hat{\beta}_{\hat{\alpha}}\left(\chi: t \zeta ; \partial^{k}(\nu)\right) & =\sum_{\theta \in \mathscr{G}} \hat{\alpha}(\theta)\left(\alpha_{\theta}, \theta_{\alpha, t \zeta, k}^{F}\right) \\
& =\sum_{\theta \in \mathscr{O}} \hat{\alpha}(\theta) \sum_{\theta^{\prime} \in \mathscr{G}} C\left(\theta_{\chi, t \zeta, k}^{F}: \theta^{\prime}\right)\left(\alpha_{\theta}, \theta^{\prime}\right) \\
& =\sum_{\theta \in \mathscr{G}} C\left(\theta_{\chi, t \zeta, k}^{F}: \theta\right) \hat{\alpha}(\theta)=\hat{\alpha}\left(\chi: t \zeta ; \partial^{k}(\nu)\right) .
\end{aligned}
$$

Recalling that $\theta_{\omega} \in \mathscr{B}$ for all $\omega \in \hat{G}^{2}$ we obviously have (2), i.e.,

$$
\widehat{\beta}_{\hat{\alpha}}\left(\theta_{\omega}\right)=\sum_{\theta \in \mathscr{\sigma}} \hat{\alpha}(\theta)\left(\alpha_{\theta}, \theta_{\omega}\right)=\hat{\alpha}\left(\theta_{\omega}\right) \text {. }
$$

Proposition 2. Let $\alpha \in C_{c}^{\infty}(G: F)$, and $\alpha_{0}=\alpha-\beta_{\hat{\alpha}}$. Then $\phi_{\hat{\alpha}_{0}} \in$ $C_{c}^{\infty}(G: F)$ and hence $\phi=\phi_{\hat{\alpha}_{0}}+\beta_{\hat{\alpha}} \in C_{c}^{\infty}(G: F)$. Furthermore, $\hat{\phi}(\chi: \nu)=$ $\hat{\alpha}(\chi: \nu)$ for all $\chi \in \hat{M}, \nu \in \mathscr{F}_{c}$.

Proof. The proof that $\phi_{\hat{\alpha}_{0}} \in C_{c}^{\infty}(G: F)$ follows from the observation that for all $\chi \in \hat{M}, t \in W(A), \zeta \in V, 0 \leqq k \leqq 0_{t}(\zeta)-1$ we have 
by (1) of Proposition 1 that,

$$
\widehat{\alpha}_{0}\left(\chi: t \zeta ; \partial^{k}(\nu)\right)=0 \text {. }
$$

By Johnson's theorem (cf. [15]) $\phi_{\hat{\alpha}_{0}}(a)($ for $\alpha(\log a) \gg 0$ ) can be expressed in terms of the residues of

$$
\Phi(\nu: a) C_{\bar{Q} \mid \bar{Q} t}(1: t \nu) \cdot t G(\chi: t \nu) \psi_{E_{F}(\chi)}(1: 1: 1) \quad(t \in W(A)) .
$$

Arguing as in the corollary to Proposition 7.2 of [15] leads to the compactness of the support of $\phi_{\hat{\alpha}_{0}}$.

For the last part we note that by (3) we have

$$
\hat{\phi}(\chi: \nu)=\hat{\phi}_{\hat{\alpha}_{0}}(\chi: \nu)+\widehat{\beta}_{\hat{\alpha}}(\chi: \nu)=\hat{\alpha}_{0}(\chi: \nu)+\widehat{\beta}_{\hat{\alpha}}(\chi: \nu)=\hat{\alpha}\left(\chi_{: \nu}\right) .
$$

I.3. The characterization of the invariant transform. Let notation be as in the preceding sections. For $L: C(G) \rightarrow C$ such that $L\left(\chi_{: \nu}\right)=L\left(\theta_{\chi, \nu}\right)$ is a $C^{\infty}$-function of $\nu$ for all $\chi \in \hat{M}$ let us define for $u \in \mathscr{S}\left(\mathscr{F}_{c}\right), \alpha \in \boldsymbol{R}$,

$$
\nu_{u, \alpha}^{p}(L)=\sup (1+|\nu|)^{\alpha}|\mathscr{L}(\chi: \nu ; u)|
$$

where the sup is taken over $\hat{M} \times \operatorname{Int} \mathscr{F}_{c}(2 / p-1)$.

Let

$$
C^{p}(G)=\left\{\theta_{\chi, \nu}: \chi \in \hat{M}, \nu \in \operatorname{Int} \mathscr{F}_{c}\left(\frac{2}{p}-1\right)\right\} \cup\left\{\theta_{\omega}: \omega \in \hat{G}^{2}\right\} \cup \mathscr{B}_{p} .
$$

Definition 1. Let $\mathscr{C}^{p}(C(G): F)_{0}$ denote the linear space of all complex valued functions defined on $C^{p}(G)$ having the following properties:

(1) for each $\chi \in \hat{M}$ the function $L(\chi)$ is holomorphic on Int $\mathscr{F}_{c}(2 / p-1)$

(2) $L(s \chi: s \nu)=L(\chi: \nu)(s \in W(A))$

(3) $L(\chi: \nu) \equiv 0$ if $\chi \notin \widehat{M}(F)$

(4) $L(\omega)=0$ if $\omega \notin \widehat{G}^{2}(F)$

(5) $\nu_{u, \alpha}^{p}(L)<\infty$ for all $u \in \mathscr{S}\left(\mathscr{F}_{c}\right), \alpha \in \boldsymbol{R}$.

Note that by the uniform continuity of $\nu \rightarrow L(\chi: \nu ; u), L(\chi: \nu ; u)$ can be extended to a continuous function on $\mathscr{F}_{c}(2 / p-1)$ which we again denote by $L(\chi: \nu ; u)$.

Definition 2. Let $\mathscr{C}^{p}(C(G): F)$ denote the subspace of functions $L \in \mathscr{C}^{p}(C(G): F)_{0}$ which satisfy the additional condition,

$$
L\left(\chi: t \zeta ; \partial^{k}(\nu)\right)=\sum_{\theta \in \mathscr{G}_{p}} C_{p}\left(\theta_{\chi, t,, k}: \theta\right) L(\theta)
$$

for all $\chi \in \hat{M}, t \in W(A), \zeta \in V_{p}$ (cf. the remark preceding the defini- 
tion), and $0 \leqq k \leqq 0_{t}(\zeta)-1$. Here if $\theta=\theta_{x^{\prime}, t^{\prime}, \zeta^{\prime}, k^{\prime}} \in \mathscr{B}_{p}$ with $k^{\prime}>0$ then by $L(\theta)$ we intend the $k^{\prime}$ th derivative of $\nu \rightarrow L\left(\theta_{x^{\prime}, \nu}\right)$ evaluated at $\nu=t^{\prime} \zeta^{\prime}$.

We give $\mathscr{C}^{p}(C(G): F)$ the topology generated by the following seminorms; let $L \in \mathscr{C}^{p}(C(G): F)$ and let $u \in \mathscr{S}\left(\mathscr{F}_{c}\right), \alpha \in \boldsymbol{R}$ and set

$$
\mu_{\alpha, r}^{p}(L)=\nu_{u, \alpha}^{p}(L)+\left(\sum_{\omega \in \hat{G}^{2}}|L(\omega)|^{2}\right)^{1 / 2} .
$$

Proposition 1. The map $\alpha \rightarrow \hat{\alpha}$ is a continuous map of $\mathscr{C}^{p}(G: F)$ into $\mathscr{C}^{p}(C(G): F)$.

Proof. The fact that $\hat{\alpha}$ is defined on $\hat{M} \times \operatorname{Int} \mathscr{F}_{c}(2 / p-1)$ and on $\theta_{\omega}\left(\omega \in \hat{G}^{2}\right)$ together with the holomorphy of $\hat{\alpha}(\chi)$ on Int $\mathscr{F}_{c}(2 / p-1)$ all follow from the corresponding properties of the transform $\alpha \rightarrow$ $\mathscr{F}_{H}(\alpha)$ (cf. [15]) and the fact that $\hat{\alpha}(\chi: \nu)=\operatorname{tr} \mathscr{F}_{H}(\alpha)\left(\chi_{: \nu}\right)$. Property (5) follows from the preceding remark together with the observation that the trace map is obviously continuous and $\alpha \rightarrow \mathscr{F}_{H}(\alpha)$ is continuous (cf. [15]). This latter comment shows moreover that given $u \in \mathscr{S}\left(\mathscr{F}_{c}\right), \alpha \in \boldsymbol{R}$, there exists $\eta$, a continuous seminorm on $\mathscr{C}^{p}(G: F)$, such that $\nu_{u, \alpha}^{p}(\widehat{\beta}) \leqq \eta(\beta)\left(\beta \in \mathscr{C}^{p}(G: F)\right)$. The condition of Definition 2 is obvious from (i) of $\S$ I.1.

Finally, the continuity follows on noting that properties (2) through (4) are obvious for $\hat{\alpha}\left(\alpha \in \mathscr{C}^{p}(G: F)\right)$ and hence as $\left|\widehat{G}^{2}(F)\right|<$ $\infty$ there exists $\eta^{\prime}$ a continuous seminorm on $\mathscr{C}^{p}(G: F)$ such that

$$
\left.\left.\left|\sum_{\omega \in \hat{G}^{2}}\right| \hat{\alpha}(\omega)\right|^{2}\right|^{1 / 2} \leqq \eta^{\prime}(\alpha) \quad\left(\alpha \in \mathscr{C}^{p}(G: F)\right) .
$$

The continuity is now obvious from the first part of the proof.

THEOREM 1. The map $\alpha \rightarrow \widehat{\alpha}$ is surjective.

Proof. Let $L \in \mathscr{C}^{p}(C(G): F$ ) and define (cf. $\S$ I.2)

$$
\beta_{L}(x)=\sum_{\theta \in \mathscr{C}_{p}} L(\theta) \alpha_{\theta}(x) \text {. }
$$

An easy computation using Proposition 1 of $\S$ I.1 shows that $\widehat{\beta}_{L}\left(\chi_{:} t \zeta ; \partial^{k}(\nu)\right)=L\left(\chi: t \zeta ; \partial^{k}(\nu)\right)$ and $\widehat{\beta}_{L}(\omega)=L(\omega)\left(\omega \in \hat{G}^{2} \mid \hat{G}^{p}\right)$. In particular if we put

$$
L_{0}=L-\widehat{\beta}_{L}
$$

and set (cf. (2) of $\S I .2$ for the definitions of $\phi_{L}$ )

$$
\psi_{L_{0}}(x)=\phi_{L_{0}}(x)+\sum_{\omega \in \hat{G}^{2}} d(\omega) d_{F}(\omega)^{-1} L_{0}(\omega) \theta_{\omega}^{F}(x)
$$


then reasoning as in [15] we see that $\psi_{L_{0}} \in \mathscr{C}^{p}(G: F)$. Hence

$$
\psi_{L}(x)=\psi_{L_{0}}(x)+\beta_{L}(x) \in \mathscr{C}^{p}(G: F) .
$$

But then

$$
\begin{aligned}
\hat{\psi}_{L}(\chi: \nu) & =\hat{\phi}_{L_{0}}\left(\chi_{:} \nu\right)+\hat{\beta}_{L}(\chi: \nu) \\
& =L(\chi: \nu)
\end{aligned}
$$

and if $\omega \in \hat{G}^{2} / \hat{G}^{p}$

$$
\hat{\psi}_{L}(\omega)=\widehat{\beta}_{L}(\omega)=L(\omega) .
$$

Whereas if $\omega \in \widehat{G}^{p}$ then as $\widehat{\beta}_{L}(\omega)=0$ (i.e., $\left(\alpha_{\theta}, \theta_{\omega}\right)=0$ if $\omega \in \widehat{G}^{p}$ )

$$
\hat{\psi}_{L}(\omega)=\hat{\psi}_{L_{0}}(\omega)=\sum_{\omega^{\prime} \in \hat{G}^{2}} d\left(\omega^{\prime}\right) d_{F}\left(\omega^{\prime}\right)^{-1} L_{0}\left(\omega^{\prime}\right)\left(\theta_{\omega^{\prime}}^{F}, \theta_{\omega}^{F}\right) .
$$

Again using the fact that $\widehat{\beta}_{L}(\omega)=0\left(\omega \in \hat{G}^{p}\right)$ and the orthogonality properties of discrete series we obtain (cf. Lemma 1, pg. 93 of [5])

$$
\hat{\psi}_{L}(\omega)=d(\omega) d_{F}(\omega)^{-1} L(\omega)\left(\theta_{\omega}^{F}, \theta_{\omega}^{F}\right)=L(\omega) .
$$

II.1. Characterization of $F_{f}$. We shall make in this section some further assumptions on $G$; further in the case of equal rank we shall pick a compact Cartan subgroup $B \subset K$, and from it construct $H$. This construction will give us an explicit Cayley transform of $\mathfrak{b}_{c}$ onto $\mathfrak{h}_{c}$. We shall then show how to change the domain of $\hat{f}$ from $C^{p}(G)$ to $L_{B} \cup \hat{H}_{k} \times a_{c}^{*} \cup \mathscr{B}_{p}$. This change actually extends the domains of these functions as well as we now explain. It is known that $\hat{M} \cong \hat{H}_{K}^{\prime} / W_{1}$ where $W_{1}=W\left(\mathfrak{m}_{c}, \mathfrak{h}_{k c}\right)\left(\mathfrak{m}=\operatorname{cent}_{k}(\mathfrak{a})\right)$ whereas in $\S \mathrm{I}, \mathfrak{h}=\mathfrak{h}_{k}+\mathfrak{a}, A=\operatorname{expa,}\left(L A\left(H_{K}\right)=\mathfrak{h}_{k}\right), \hat{H}_{K}^{\prime}$ denotes the set of regular elements of $\hat{H}_{K}$ (we shall make exact definitions below) and $\hat{H}_{K}^{\prime} / W_{1}$ denotes the equivalence classes of $\hat{H}_{K}^{\prime}$ under $W_{1}$. Also it was pointed out in $\S$ I that $\hat{G}^{2} \cong \mathscr{L}_{B}^{\prime} / W$; hence with these new definitions $\hat{f}$ will be defined for both singular and regular elements of $\mathscr{L}_{B}$ and $\hat{H}_{K}$ respectively. This is necessitated because one must use all the characters of $\hat{B}$ and $\hat{H}_{K}$ in order to expand functions on $B$ and $H_{k}$.

Let us assume that we have a group of equal rank; let $B$ be a compact Cartan subgroup, $\mathfrak{b}$ its Lie algebra. Fix a singular imaginary root $\beta$ of the pair $(\mathfrak{g}, \mathfrak{b})$ and a point $\Gamma \in \mathfrak{b}$ such that $\pm \beta$ are the only roots of the pair $(\mathfrak{g}, \mathfrak{b})$ which vanish at $\Gamma$. Let $\mathfrak{g}=\operatorname{Cent}_{\mathfrak{g}}(\Gamma)$, and $\mathfrak{c}_{\Gamma}, \mathfrak{l}_{\Gamma}$ the center and derived algebra respectively of $\mathfrak{g}_{\Gamma}$.

$\mathfrak{l}_{\Gamma}$ is isomorphic over $\boldsymbol{R}$ to $s \zeta(2, \boldsymbol{R})$, and we may select a basis $H^{*}, X^{*}, Y^{*}$ for $\mathfrak{l}$ (over $R$ ) such that $\left[H^{*}, X^{*}\right]=2 X^{*},\left[H^{*}, Y^{*}\right]=-$ $2 Y^{*},\left[X^{*}, Y^{*}\right]=H^{*}$. Then $\mathfrak{b}=\boldsymbol{R}\left(X^{*}-Y^{*}\right)+\mathfrak{l}_{\Gamma}$ and $\mathfrak{h}=\boldsymbol{R} H^{*}+\mathfrak{c}_{F}$ form a complete set of nonconjugate Cartan subalgebras of $\mathfrak{g}$. Put 
$\mu=\exp \left[V \overline{-1}(\pi / 4)\left(X^{*}+Y^{*}\right)\right] \in G_{c}$. Then $\mathfrak{b}_{c}^{\mu}=\mathfrak{h}_{c}$, if $\alpha=\beta^{\mu}$, we have $\alpha\left(H^{*}\right)=2$ and $\alpha$ vanishes identically on $c_{\Gamma}$; we shall thus again denote by $\alpha$ the restriction of $\alpha$ to $a=R H^{*}$. Order the space of real linear functionals $\lambda$ on $R H^{*}+\sqrt{-1} c_{\Gamma}$ by requiring that $\lambda>0$ whenever $\lambda\left(H^{*}\right)>0$. We then obtain a set of positive roots for the pair $(\mathfrak{g}, \mathfrak{b})$ by requiring that the $\mu$-transform of such a root be positive when considered as a root of $(\mathfrak{g}, \mathfrak{h})$.

Let $H$ be the Cartan subgroup of $G$ associated with $\mathfrak{h}$, and let $H^{0}$ be the identity component of $H$. Then, setting $H_{K}=H \cap K$, $H_{K}^{0}=H^{0} \cap K$ and $A=\left\{\operatorname{expt} H^{*}: t \in R\right\}$, we have $H=H_{K} A$, and $H^{0}=$ $H_{K}^{0} A$. Put $Z(A)=K \cap \exp \left\{i R H^{*}\right\}$. Then $Z(A)=\{1, \gamma\}$ is a group of order two with $\gamma=\exp \left[\pi\left(X^{*}-Y^{*}\right)\right]=\exp \left(i \pi H^{*}\right) \neq 1$. We have $H_{K}=Z(A) H_{K}^{0}$.

Set $\mathfrak{b}_{1}=\mathfrak{c}_{\Gamma}, \mathfrak{b}_{2}=\boldsymbol{R}\left(X^{*}-Y^{*}\right)$ and let $B_{1}, B_{2}$ be the analytic subgroups of $B$ corresponding to $\mathfrak{b}_{1}$ and $\mathfrak{b}_{2}$ respectively. $B_{1}$ and $B_{2}$ are compact and $B_{1} \cap B_{2} \subset Z(A)$. Since $H_{K}=B_{1} \cup \gamma B_{1},\left(B_{1}=H_{K}^{0}\right)$, it follows that $H_{K}$ has one or two connected components according to whether $\gamma$ lies in $B_{1} \cap B_{2}$ or not. If $M=\operatorname{Cent}_{K}(A), M^{0}$ its identity component, then $M=M^{0} \cup \gamma M^{0}$.

If no simple factor of $G$ is isomorphic to $S L(2, R)$, it follows from the classification of real rank one groups that $M$ is connected, or equivalently that $B_{1} \cap B_{2}=\{1, \gamma\}$. In this case $B_{1}=H_{k}$ is a maximal torus in $M$. As in [11] we shall now assume that $M$ is connected.

Choose a normalized invariant measure $d_{G / B}(\dot{x})$ as in [19] v. II. If we choose a Haar measure $d_{B}(b)$ on $B$ normalized so that the volume of $B$ is one, then a Haar measure $d x$ on $G$ is fixed by the formula

$$
\int_{G} f(x) d x=\int_{G / B} \int_{B} f(x b) d b d_{G / B}(\dot{x}) \quad\left(f \in C_{c}(G)\right) .
$$

Let $d_{A}(h)$ be the Haar measure on $A$ which is the transport via the exponential map of the canonical Haar measure on the Lie algebra a associated with the Euclidean structure derived from the Killing form of $\mathrm{g}$. Since $A=\left\{\operatorname{expt} H^{*}: t \in \boldsymbol{R}\right\}$ we have $d_{A}(h)=c_{A} d t$ where $c_{A}$ is a positive constant and $d t$ is normalized Lebesgue measure on $\boldsymbol{R}$. Normalize Haar measure $d_{H_{K}}(h)$ on $H_{K}$ so that the volume of $H_{K}$ is one. Now a Haar measure $d_{H}(h)$ on $H$ is fixed by the formula $d_{H}(h)=d_{H_{K}}\left(h_{1}\right) d_{A}\left(h_{2}\right)\left(h=h_{1} h_{2}, h_{1} \in H_{K}, h_{2} \in A\right)$. A $G$-invariant measure $d_{G / H}(\dot{x})$ on $G / H$ is then determined by the formula

$$
\int_{G} f(x) d x=\int_{G / H} \int_{H} f(x h) d_{H}(h) d_{G / H}(\dot{x}) \quad\left(f \in C_{c}(G)\right) .
$$


If $x \in G$ write $b^{\dot{x}}=x b x^{-1}(b \in B)$, where $\dot{x}$ denotes the image of $x$ under the canonical map of $G$ onto $G / B$; similarly let $h^{\dot{x}}=x h x^{-1}$ $(h \in H$ ) where now $\dot{x}$ is the image of $x$ under the canonical map of $G$ onto $G / H$. If $b, h$ are as above, $f \in C_{c}^{\infty}(G)$ let

$$
\begin{gathered}
F_{f}^{B}(b)=\Delta_{B}(b) \int_{G / B} f\left(b^{\dot{x}}\right) d_{G / B}(\dot{x}) \\
F_{f}^{H}(h)=\Delta_{H}(h) \varepsilon_{R}^{H}(h) \int_{G / H} f\left(h^{\dot{x}}\right) d_{G / H}(\dot{x}),
\end{gathered}
$$

(for definitions of $\Delta_{B}, \Delta_{H}$, and $\varepsilon_{R}^{H}$ one may refer to [19]). By Weyl's integration formula we have

$$
\begin{aligned}
& \int_{G} f(x) d x \\
& \quad=|W(G / B)|^{-1} \int_{B} \overline{A_{B}(b)} F_{f}^{B}(b) d_{B}(b)+|W(G / H)|^{-1} \int_{H} \overline{A_{H}(h)} \varepsilon_{R}^{H}(h) F_{f}^{H}(h) d_{H}(h) .
\end{aligned}
$$

For $f \in C_{c}^{\infty}(G)$ the invariant integrals have the following properties;

(1) $F_{f}^{B}(w b)=\operatorname{det}(w) F_{f}^{B}(b)\left(w \in W(G / B), b \in B^{\prime}\right)$

(2) $\quad F_{f}^{H}\left(h_{1} h_{2}\right)=F_{f}^{H}\left(h_{1} h_{2}^{-1}\right)\left(h_{1} \in H_{K}, h_{2} \in A\right)$

(3) $F_{f}^{H}(w h)=\operatorname{det}(w) F_{f}^{H}(h)\left(h \in H^{\prime}, w \in W\left(M / H_{K}\right)\right)$.

Further, it is known that $F_{f}^{B} \in C^{\infty}\left(B^{\prime}\right)$ (here $G^{\prime}$ denotes the set of regular elements of $G$ and for any subset $L$ of $G, L^{\prime}=L \cap G^{\prime}$ ) and in general $F_{f}^{B}$ does not extend to a $C^{\infty}$ function on all of $B$. The function $F_{f}^{H} \in C^{\infty}\left(H^{\prime}\right)$ and extends to a compactly supported $C^{\infty}$ function on all of $H$ since $(\mathfrak{g}, \mathfrak{h})$ has no singular imaginary roots.

Recall that $\hat{B} \cong \mathscr{L}_{B}$. The Weyl group $W(\mathfrak{g}, \mathfrak{b})$ acts on $\mathscr{L}_{B}$ and hence on $\hat{B}$ by the prescription

$$
w \Lambda(H)=\Lambda\left(w^{-1} H\right), w \xi_{\Lambda}(h)=\xi_{w 1}(h) \quad\left(H \in b, \Lambda \in \mathscr{L}_{B}\right)
$$

(here $\left.\xi_{\Lambda}(\exp H)=e^{\Lambda(H)}\right)$.

We say that $\Lambda \in \mathscr{L}_{B}$ is regular if $w \Lambda \neq \Lambda$ for all $w \neq 1$ in $W\left(\mathfrak{g}_{c}, \mathfrak{b}_{c}\right)$; otherwise we say $\Lambda$ is singular. The set of regular $A$ will be denoted by $\mathscr{L}_{B}^{\prime}$ and the set of singular $\Lambda$ by $\mathscr{L}_{B}^{s}$. The character $\xi_{A}$ is called regular or singular accordingly.

To each $\Lambda \in \mathscr{L}_{B}$, there is associated a central eigendistribution $\theta_{A}$ on $G$ characterized uniquely by certain properties (cf. [19]). $\theta_{A}$ is locally summable on $G$ and analytic on $G^{\prime}$. We have

$$
\theta_{A}(b)=\Delta_{B}(b)^{-1} \sum_{w \in W(G / B)} \operatorname{det}(w) \xi_{w A}(b), b \in B^{\prime} .
$$

If $\Lambda \in \mathscr{L}_{B}^{s}$ and if $\Lambda$ is fixed by a nontrivial element of $W(G / B)$, then $\theta_{1} \equiv 0$ on $B^{\prime}$. 
For $\Lambda \in \mathscr{L}_{B}^{\prime}$, put $s=1 / 2 \operatorname{dim}(G / K)$ and $\varepsilon(\Lambda)=\operatorname{sgn}\left\{\Perp_{\beta \in P_{B}}(\Lambda, \beta)\right\}$ where $P_{B}$ denotes a set of positive roots of $(\mathfrak{g}, \mathfrak{b})$. Then

$$
\theta_{\Lambda}=(-1)^{8} \varepsilon(\Lambda) \theta_{\Lambda}
$$

is a character of a representation $\omega(\Lambda)$ in the discrete series for $G$ and all discrete series characters are obtained in this way.

For $\lambda \in \hat{H}_{K}$, the unitary character group of $H_{K}$, denote by $\log \lambda$ the linear function on $\mathfrak{b}_{1}=\mathfrak{h}_{k}$ defined by

$$
\lambda(\exp H)=e^{\langle H, \log \lambda\rangle} \quad\left(H \in \mathfrak{b}_{1}\right) .
$$

Let $P_{I}^{+}$be the set of positive imaginary roots of the pair $(\mathfrak{g}, \mathfrak{h})$, and $W_{1}$ be the subgroup of $W\left(\mathfrak{g}_{c}, \mathfrak{h}_{c}\right)=W$ which is generated by the Weyl reflections associated with elements of $P_{I}^{+} . W_{1}$ may be identified with the Weyl group of $W\left(\mathfrak{m}_{c}, \mathfrak{h}_{k c}\right)$. An element $\lambda \in \hat{H}_{K}$ is called regular if $w \lambda \neq \lambda$ for all $w \neq 1$ in $W_{1}$ and singular otherwise. If $\lambda \in \hat{H}_{K}$ is singular put $\varepsilon(\lambda)=1$, and if $\lambda$ is regular put

$$
\varepsilon(\lambda)=\operatorname{sgn}\left\{\prod_{\alpha \in P_{I}^{+}}(\log \lambda, \alpha)\right\} .
$$

The unitary character group $\hat{A}$ of $A$ is isomorphic to $R$ and, for $\nu \in \boldsymbol{R}$, we define the corresponding unitary character on $A$ by

$$
h^{i \nu}=e^{i \nu}(\log h) \quad(h \in A) .
$$

If $\phi \in C_{c}^{\infty}(A)$ define its Fourier transform by

$$
\hat{\phi}(\lambda: \nu)=(2 \pi)^{-1 / 2} \int_{H_{K}} \int_{A} \lambda\left(h_{1}\right) h_{2}^{i \nu} \dot{\phi}\left(h_{1} h_{2}\right) d_{H_{K}}\left(h_{1}\right) d_{A}\left(h_{2}\right) \quad\left(\lambda \in \hat{H}_{K}, \nu \in \boldsymbol{R}\right) .
$$

If $\lambda$ is singular it follows from (3) that $\hat{\phi}(\lambda: \nu) \equiv 0$. We have the following inversion formula for all $\phi$ as above;

$$
\phi\left(h_{1} h_{2}\right)=C_{A}^{-1}(2 \pi)^{-1 / 2} \sum_{\lambda \in \hat{H}_{K}} \overline{\lambda\left(h_{1}\right)} \int_{-\infty}^{\infty} e^{-i \nu\left(\log h_{2}\right)} \hat{\phi}(\lambda: \nu) d \nu,
$$

where $d \nu$ is normalized Lebesgue measure on $\boldsymbol{R}$.

The following now gives the relation between the Fourier transform of the invariant integral $F_{f}^{H}$ and the invariant transform of $f$ with respect to principal series characters. Suppose $\lambda \in \hat{H}_{K}$ is a regular character, $\nu \in \hat{A}$, and $r_{I}=\left|P_{I}^{+}\right|$. Then the distribution,

$$
\theta_{\lambda, \nu}(f)=(2 \pi)^{1 / 2}(-1)^{r_{I}} \varepsilon(\lambda) \hat{F}_{f}^{H}(\lambda: \nu) \quad\left(f \in C_{c}^{\infty}(G)\right)
$$

is the character of a principal series representation which we denote by $\pi_{x(\lambda), \nu}$. We have then in the notation of $\S I$ that

$$
\theta_{\lambda, \nu}=\theta_{\chi(\lambda), \nu} \quad\left(\forall \text { regular } \lambda \text { in } \hat{H}_{K}\right) .
$$


If $\lambda$ is singular define $\theta_{\lambda, \nu}$ by the right hand side of (4). Of course $\theta_{\lambda, \nu} \equiv 0$ for singular $\lambda$. It follows from the general theory of finite dimensional representations of semisimple Lie groups that

$$
\theta_{s \lambda, 2}=\theta_{\lambda, \nu} \quad\left(s \in W_{1}\right)
$$

where if $\lambda(H)=e^{\langle H, \log \lambda\rangle}$ then $s \lambda(H)$ is defined to be $e^{\langle H, s \log \rangle}$.

Note also that from (2) we have

$$
\theta_{\lambda, \nu}=\theta_{\lambda,-\nu} \quad\left(\lambda \in \hat{H}_{K}, \nu \in \boldsymbol{R}\right) .
$$

If $\phi \in L^{1}(B)$ define

$$
\hat{\phi}(\Lambda)=\int_{B} \xi_{\Lambda}(b) \phi(b) d b \quad\left(\Lambda \in L_{B}\right) .
$$

We shall now need the following result.

THEOREM 1. ([11] Theorem 3.19.). Suppose that $b_{0} \in B^{\prime}$. For $w \in W(G / B)$, we write $w^{-1} b_{0}=b_{1}(w) b_{2}(w)$ where $b_{1}(w) \in B_{1}$ and $b_{2}(w)=$ $\exp \left(\theta_{w}\left(X^{*}-Y^{*}\right)\right) \in B_{2}$. Then, if $f \in C_{c}^{\infty}(G)$ we have

$$
F_{f}^{B}\left(b_{0}\right)=(-1)^{r} \sum_{A \in L_{B}} \theta_{\Lambda}(f) \overline{\xi_{A}\left(b_{0}\right)}+I_{f}\left(b_{0}\right)
$$

where

$$
I_{f}\left(b_{0}\right)
$$

$$
\begin{aligned}
= & \frac{1}{2}(-1)^{r_{I}}\left|W\left(\mathfrak{g}_{c}, \mathfrak{h}_{c}\right)\right|^{-1} \sum_{w \in W(G / B)} \operatorname{det}(w) \sum_{\lambda \in \hat{H}_{K}} \varepsilon(\lambda) \\
& \times\left\{\overline{\lambda\left(b_{1}(w)\right)} \int_{-\infty}^{\infty} \theta_{\lambda, \nu}(f) \eta_{1}\left(\nu: \theta_{w}\right) d \nu+\overline{\lambda\left(\gamma b_{1}(w)\right)} \int_{-\infty}^{\infty} \theta_{\lambda, \nu}(f) \eta_{2}\left(\nu: \theta_{w}\right) d \nu\right\}
\end{aligned}
$$

where

$$
\begin{aligned}
& \eta_{1}\left(\nu: \theta_{w}\right)=\sinh \left(\nu\left(\theta_{w} \mp \pi\right)\right) / \sinh (\nu \pi) \\
& \eta_{2}\left(\nu: \theta_{w}\right)=\sinh \left(\nu \theta_{w}\right) / \sinh (\nu \pi) .
\end{aligned}
$$

Here in the definition of $\eta_{1}$ we choose the minus sign if $0<\theta_{w}<\pi$ for all $w \in W(G / B)$ and the plus sign otherwise.

Now for $f \in \mathscr{C}^{p}(G: F)$ (cf. $\S$ I) let us put for $\Lambda \in \mathscr{L}_{B}, \lambda \in \hat{H}_{K}, \nu \in \boldsymbol{R}$

$$
\hat{f}(\Lambda)=\theta_{\Lambda}(\check{f}), \hat{f}(\lambda: \nu)=\theta_{\lambda, \nu}(\check{f}) \text {. }
$$

Henceforth we shall assume that the invariant transform is defined on $\mathscr{D}_{p}=\mathscr{L}_{B} \cup \hat{H}_{K} \times \mathscr{F}_{c}(2 / p-1) \cup \mathscr{B}_{p}(0<p<2)$. Using the isomorphisms $\hat{G}^{2} \cong \mathscr{L}_{B}^{\prime} / W(G / B)$ and $\hat{M} \cong \hat{H}_{K}^{\prime} / W_{1}$ it is not hard to give the following characterization of the invariant transform of $\mathscr{C}^{p}(G: F)$. First we need one fact; by [1] there exists for each 
$\Lambda \in \mathscr{L}_{B}^{s}$ a distribution $S_{A}$ living on $\mathscr{L}_{B}^{\prime} \cup \hat{H}_{K}^{\prime} \times \mathscr{F}$ such that

$$
\theta_{\Lambda}(f)=S_{\Lambda}\left((f)^{\wedge}\right) \quad(f \in \mathscr{C}(G)) .
$$

That is, the values of $\hat{f}$ on $\mathscr{L}_{B}^{s}$ are determined by its values on the characters of the tempered representations of $G$. It follows from this that if $f \in \mathscr{C}^{p}(G: F)$ then

$$
\hat{f}(\Lambda)=S_{\Lambda}(\hat{f}) .
$$

Let now $\mathscr{C}^{p}(C(G): F)$ be defined as those functions $L: \mathscr{D}_{p} \rightarrow C$ satisfying properties of Definitions 1 and 2 of $\S I .3$ subject to the changes that $\chi \in \hat{M}$ is to be replaced by $\lambda \in \hat{H}_{K}, \omega$ by $\lambda \in \mathscr{L}_{B}$, and $\widehat{M}(F), \hat{G}^{2}(F)$ are to be replaced by the sets $\hat{H}_{K}(F)$ and $\mathscr{L}_{B}(F)$ which have their obvious meaning. We must then add two further conditions reflecting $\left({ }^{*}\right)$ which we number conditions 6 and 7 .

(6) $L(\omega \lambda: \nu)=L(\lambda: \nu)\left(\omega \in W_{1}\right), L(\omega \Lambda)=L(\Lambda)(\omega \in W(G / B))$

( 7 ) $L(\Lambda)=S_{\Lambda}(L)\left(\Lambda \in \mathscr{L}_{B}^{s}\right)$.

We shall now designate the normalized orbital integrals of $f \in$ $\mathscr{C}(G)$ by $F_{f}$; the argument of $F_{f}$ will make it clear whether we are considering $F_{f}^{H}$ or $F_{f}^{B}$. Similarly when we take the Fourier transform of $F_{f}$ the arguments again will make it clear whether we are transforming on $H$ or $B$.

Proposition 1. For all $f \in \mathscr{C}^{p}(G: F)(0<p<2)$

(i ) $\hat{F}_{f}(\lambda: \nu)=(2 \pi)^{-1 / 2}(-1)^{r} I \varepsilon(\lambda)(\check{f})^{\wedge}(\lambda: \nu)\left(\lambda \in \hat{H}_{K}, \nu \in \mathscr{F}_{c}(2 / p-1)\right)$

(ii) Let

$$
{ }^{\circ} F_{f}(b)=F_{f}(b)-I_{f}(b) \quad\left(b \in B^{\prime}\right)
$$

then

$$
\left({ }^{\circ} F_{f}\right)^{\wedge}(\Lambda)=(-1)^{r}(f)^{\wedge}(\Lambda) \quad\left(\Lambda \in \mathscr{L}_{B}\right)
$$

Proof. (i) is just a reformulation of (4); (ii) follows from Theorem 1 and Fourier inversion on $B$.

Let $0<p<2$ and for $\phi: B^{\prime} \cup H \rightarrow C, \phi$ of class $C^{\infty}, u \in \mathfrak{S}, v \in \mathfrak{B}$, $n \in \boldsymbol{Z}$, and set

$$
\eta_{u, v, n}^{p}(\phi)=\sup _{H_{K^{\times}} A} e^{(2 / p-1) \rho\left(\log h_{2}\right)}\left(1+\sigma\left(h_{2}\right)\right)^{n}\left|\phi\left(h_{1} h_{2} ; u\right)\right|+\sup _{B^{\prime}}|\phi(b ; v)| .
$$

Let $\theta$ be the character of a quasi-simple representation of $G$. Set $\check{\theta}(\alpha)=\theta(\check{\alpha})\left(\alpha \in C_{c}^{\infty}(G)\right)$ and,

$$
\begin{aligned}
& \theta_{B}=[W(G / B)]^{-1} \bar{\Delta}_{B} \cdot \theta, \quad \theta_{B}^{*}=[W(G / B)]^{-1} \bar{\Delta}_{B} \cdot \check{\theta} \\
& \theta_{H}=[W(G / H)]^{-1} \bar{\Delta}_{H} \cdot \varepsilon^{H} \cdot \theta, \quad \theta_{H}^{*}=[W(G / H)]^{-1} \bar{\Delta}_{H} \cdot \varepsilon_{R}^{H} \cdot \check{\theta} .
\end{aligned}
$$

Then for all $f \in \mathscr{C}(G)$ we have 


$$
\begin{aligned}
\theta(f) & =\int_{B^{\prime}} \theta_{B}(b) F_{f}(b) d_{B}(b)+\int_{H} \theta_{H}(h) F_{f}(h) d_{H}(h) \\
& =\int_{B^{\prime}} \theta_{B}^{*}(b) F_{f}(b) d_{B}(b)+\int_{H} \theta_{H}^{*}(h) F_{f}(h) d_{H}(h) \\
& =\left(\theta_{B}^{*}, F_{f}^{\curlyvee}\right)+\left(\theta_{H}^{*}, F_{\check{f}}^{\searrow}\right) .
\end{aligned}
$$

The second line follows from; $\left|\Delta_{H}(h)\right|^{2}=\left|\Delta_{H}\left(h^{-1}\right)\right|^{2},\left|\Delta_{B}(b)\right|^{2}=\left|\Delta_{B}\left(b^{-1}\right)\right|^{2}$.

Proposition 2. Let $f \in \mathscr{C}^{p}(G: F)(0<p<2)$.

(a) $\hat{F}_{f}(\lambda)$ is a holomorphic function on $\operatorname{Int}\left(\mathscr{F}_{c}(2 / p-1)\right)$ for all $\lambda \in \hat{H}_{K}$.

(b) $\hat{F}_{f}(\lambda) \equiv 0$ if $\lambda \notin \hat{H}_{K}(F)$.

(c) $\hat{F}_{f}(s \lambda: s \nu)=\varepsilon(s \lambda) / \varepsilon(\lambda) \hat{F}_{f}(\lambda: \nu)\left(s \in W(A), \lambda \in \hat{H}_{K}, \nu \in \mathscr{F}_{c}(2 / p-1)\right)$.

(d) $\hat{F}_{f}\left(\lambda: t \zeta ; \partial^{k}(\nu)\right)=(2 \pi)^{-1 / 2}(-1)^{r} I \varepsilon(\lambda) \sum_{\theta \in \mathscr{O}_{p}} C_{p}\left(\theta_{\chi, t \zeta, k}: \theta\right)\left(\left(\theta_{B}^{*}, F_{f}\right)+\right.$ $\left.\left(\theta_{H}^{*}, F_{f}\right)\right)$ for all $t \in W(A), \zeta \in V_{p}, 0 \leqq k \leqq 0_{t}(\zeta)-1$.

(e) ${ }^{\circ} \hat{F}_{f}(\Lambda)=0$ if $\Lambda \notin \mathscr{L}_{B}(F)$.

(f) $\eta_{u, v, n}^{p}\left(F_{f}\right)<\infty$ for all $u \in \mathfrak{S}, v \in \mathfrak{B}, n \in \boldsymbol{Z}$.

Proof. Statements (a) through (e) all follow from (i) of Proposition 1 together with the corresponding properties of $\hat{f}$.

For (f) we note that

$$
F_{f}\left(h_{1} h_{2}\right)=C_{A}^{-1}(2 \pi)^{-1}(-1)^{r_{I}} \sum_{\lambda \hat{H}_{K}} \varepsilon(\lambda) \overline{\lambda\left(h_{1}\right)} \int_{-\infty}^{\infty} e^{-i \nu\left(\log h_{2}\right)}(\check{f})^{\wedge}(\lambda: \nu) d \nu .
$$

Hence, if $u \in \mathfrak{S E}$ then we can write $u=\sum_{i} \xi_{i} \eta_{i}$ where $\xi_{i} \in \mathfrak{S}_{k}$ and $\eta_{i} \in \mathfrak{A}$. From this and the fact that $\hat{f}(\lambda: \nu) \equiv 0$ for $\lambda \notin \hat{H}_{K}(F)$ we have,

$F_{f}\left(h_{1} h_{2} ; u\right)$

$$
=\text { const } \sum_{j} \sum_{\lambda \in H_{K^{\prime}}(F)} \varepsilon(\lambda) \xi_{j} \overline{(\log \lambda)} \lambda\left(h_{1}\right) \int_{-\infty}^{\infty} e^{-i \nu\left(\log h_{2}\right)} \eta_{j}(-i \nu)(\check{f})^{\wedge}(\lambda: \nu) d \nu .
$$

Using the holomorphy on $\operatorname{Int}\left(\mathscr{F}_{c}(2 / p-1)\right)$ and continuity on $\mathscr{F}_{c}(2 / p$ $-1)$ we have on letting $\rho_{p}=(2 / p-1)$ that

$$
\begin{aligned}
F_{f}\left(h_{1} h_{2} ; u\right) & \\
= & \operatorname{const} \sum_{j} \sum_{\lambda} \varepsilon(\lambda) \overline{\xi_{j}(\log \lambda)} \lambda\left(h_{1}\right) e^{-\rho_{p}\left(\log h_{2}\right)} \\
& \times \int_{-\infty}^{\infty} e^{-i \nu\left(\log h_{2}\right)} \eta_{j}\left(-\rho_{p}-i \nu\right)(\breve{f})^{\wedge}\left(\lambda:-\rho_{p}-i \nu\right) d \nu .
\end{aligned}
$$

On can easily deduce from Lemma 8.1 of [15] that the function $\nu \rightarrow(\check{f})^{\wedge}\left(\lambda:-\rho_{p}-i \nu\right)$ belongs to the Schwartz space of $\boldsymbol{R}$ for all $\lambda \in \hat{H}_{K}(F)$ and that there exists for every $n \in N$ (here we are combining the fact that the map $f \rightarrow \hat{f}$ is continuous with Lemma 8.1 ibid.) a continuous seminorm $\nu$ on $\mathscr{C}^{p}(G: F)$ such that

$$
(1+\sigma(h))^{n} \sum_{j}\left|\int_{-\infty}^{\infty} e^{-i \nu\left(\log h_{2}\right)} \eta_{j}\left(-\rho_{p}-i \nu\right)(\breve{f})^{\wedge}\left(\lambda:-\rho_{p}-i \nu\right) d \nu\right| \leqq \nu(f) .
$$


As $\left|\hat{H}_{K}(F)\right|<\infty$ then it is clear that there exists a continuous seminorm $\nu_{1}$ on $\mathscr{C}^{p}(G: F)$ such that

$$
\sup _{H_{K^{\times}}} e^{(2 / p-1) \rho\left(\log h_{2}\right)}\left(1+\sigma\left(h_{2}\right)\right)^{n}\left|F_{f}\left(h_{1} h_{2} ; u\right)\right| \leqq \nu_{1}(f) .
$$

Let $v \in \mathfrak{B}$. Then from Theorem 11 of [18] it follows that $v F_{f}$ has finite jump discontinuities on $B-B^{\prime}$; moreover, the jumps can be bounded by derivatives of $\left.F_{f}\right|_{H}$. Given the continuity of the map $f \rightarrow F_{f}$, as a map of $\mathscr{C}^{p}(G: F)$ into $C^{\infty}\left(B^{\prime}\right)$ (cf. [18] $\S 12.2$ ), and the continuity of the map $f \rightarrow F_{f}$ as a map of $\mathscr{C}^{p}(G: F)$ into $C^{\infty}(H)$ we see that there exists a continuous seminorm $\nu_{2}$ on $\mathscr{C}^{p}(G: F)$ such that

$$
\left|F_{f}(b ; v)\right| \leqq \nu_{2}(f)
$$

Hence (f) follows.

Definition 1. Let $D=B^{\prime} \cup H$ and for every $0<p<2$ let $I^{p}(D: F)$ denote the space of all functions $\phi: D \rightarrow C$ such that $\left.\phi\right|_{H I} \in$ $C^{\infty}(H),\left.\phi\right|_{B^{\prime}} \in C^{\infty}\left(B^{\prime}\right), \phi\left(h_{1} h_{2}\right)=\phi\left(h_{1} h_{2}^{-1}\right)\left(h_{1} \in H_{k}, h_{2} \in A\right), \phi(w h)=\operatorname{det}(w) \phi(h)$ $\left(h \in H, w \in W_{1}\right), \phi(w b)=\operatorname{det}(w) \phi(b)\left(w \in W(G / B), b \in B^{\prime}\right)$, and if $b \in B^{\prime}$ and,

$$
\begin{gathered}
I_{\phi}(b)=(i / 2)\left|W\left(\mathfrak{g}_{c}, \mathfrak{h}_{c}\right)\right|^{-1} \sum_{w \in W(G \mid B)} \operatorname{det}(w) \sum_{\lambda \in \hat{H}_{K}}(2 \pi)^{1 / 2} \cdot \\
\left\{\overline{\lambda\left(b_{1}(w)\right)} \cdot \int_{-\infty}^{\infty} \hat{\phi}(\lambda: \nu) \eta_{1}\left(\nu: \theta_{w}\right) d \nu+\overline{\lambda\left(\gamma b_{1}(w)\right)} \int_{-\infty}^{\infty} \hat{\phi}(\lambda: \nu) \eta_{2}\left(\nu: \theta_{w}\right) d \nu\right\}
\end{gathered}
$$

$\left(\eta_{1}, \eta_{2}\right.$ defined in Theorem 1$)$, and

$$
{ }^{\circ} \phi(b)=\dot{\phi}(b)-I_{\phi}(b)
$$

then

$$
{ }^{\circ} \hat{\phi}(\Lambda)=0 \text { if } \Lambda \notin \mathscr{L}_{B}(F) .
$$

Further we require that $\phi$ satisfy properties (a) through (d) and property $(f)$ of Proposition 2 (with $\phi$ replacing $F_{f}$ ).

We topologize $I^{p}(D: F)$ using the seminorms $\eta_{u, v, n}^{p}$. As a result of Proposition 2 and its proof we have the following.

Corollary. The map $f \rightarrow F_{f}$ is a continuous map of $\mathscr{C}^{p}(G: F)$ into $I^{p}(D: F)(0<p<2)$.

We now come to the principal result of $\S$ II.

THEOREM 2. The map $f \rightarrow F_{f}$ is a continuous surjection of $\mathscr{C}^{p}(G: F)$ onto $I^{p}(D: F)$. 
Proof. All that remains to be shown is the surjectivity. Let $\phi \in I^{p}(D: F)$ and put

$$
\begin{array}{ll}
L(\lambda: \nu)=(2 \pi)^{1 / 2}(-1)^{r_{I}} \varepsilon(\lambda) \hat{\phi}(\lambda: \nu) & \left(\lambda \in \hat{H}_{K}, \nu \in \mathscr{F}_{c}(2 / p-1)\right) \\
L(\theta)=\left(\theta_{B}^{*}, \phi\right)+\left(\theta_{H}^{*}, \phi\right) & \left(\theta \in B_{p}\right) \\
L(\Lambda)=(-1) \hat{\phi}(\Lambda) & \left(\Lambda \in \mathscr{L}_{B}\right)
\end{array}
$$

here ${ }^{\circ} \phi$ is defined as in (10).

As $\phi\left(h_{1} h_{2}\right)$ is of exponential type in $h_{2}$ for each $h_{1} \in H_{K}$ it follows from the classical Paley-Wiener theorem applied to the vector group $A$ that for each $\lambda \in \hat{H}, \nu \rightarrow L\left(\chi_{:}: \nu\right)$ extends to a holomorphic function on $\operatorname{Int}\left(\mathscr{F}_{c}(2 / p-1)\right)$ and that for each $u \in \mathscr{S}\left(\mathscr{F}_{c}\right), \alpha \in \boldsymbol{R}$

$$
\sup |L(\lambda: \nu ; u)|(1+|\nu|)^{\alpha}<\infty
$$

where the sup is taken over $(\lambda, \nu) \in \hat{H}_{K} \times \operatorname{Int}\left(\mathscr{F}_{i}(2 / p-1)\right)$. Further, since $\varepsilon(w \lambda)=\varepsilon(\lambda)\left(w \in W_{1}, \lambda \in \hat{H}_{K}\right)$ it follows from property (c) of Proposition 2 that,

$$
\begin{aligned}
& L(w \lambda: \nu)=L(\lambda: \nu) \text { and } L(s \lambda: s \nu)=L(\lambda: \nu) \\
& L(\lambda: \nu) \equiv 0\left(\lambda \notin \hat{H}_{K}(F)\right) \text { and } L(\Lambda)=0\left(\Lambda \notin \mathscr{L}_{B}(F)\right) .
\end{aligned}
$$

We have for $t \in W(A), \zeta \in V_{p}, \lambda \in \hat{H}_{K}, 0 \leqq k \leqq 0_{t}(\zeta)-1$ that

$$
\begin{aligned}
L\left(\chi: t \zeta ; \partial^{k}(\nu)\right) & =(2 \pi)^{1 / 2}(-1)^{r_{I} \varepsilon}(\lambda) \hat{\phi}\left(\lambda: t \zeta ; \partial^{k}(\nu)\right) \\
& =\sum_{\theta \in \mathscr{O}_{p}} C_{p}\left(\theta_{\chi, t \zeta, k}: \theta\right)\left(\left(\theta_{B}^{*}, \phi\right)+\left(\theta_{H}^{*}, \phi\right)\right) \\
& =\sum_{\theta \in \mathscr{F}_{p}} C_{p}\left(\theta_{\chi, t \zeta, k}: \theta\right) L(\theta) .
\end{aligned}
$$

It follows from Theorem 1 of $\S$ I.3 that as $L \in \mathscr{C}^{p}(C(G): F)$ there exists $f \in \mathscr{C}^{p}(G: F)$ such that $\hat{f}=L$. Hence by Proposition 1 ,

$$
\hat{F}_{f}(\lambda: \nu)=(2 \pi)^{-1 / 2}(-1)^{r} \varepsilon \varepsilon(\lambda) \hat{f}(\lambda: \nu)=\hat{\phi}(\lambda: \nu) .
$$

Therefore

$$
F_{\breve{f}}(h)=\phi(h) \quad(h \in H) .
$$

We also have

It follows that

$$
{ }^{\circ} \hat{F}_{\breve{f}}(\Lambda)=(-1)^{r} \hat{f}(\Lambda)={ }^{\circ} \hat{\phi}(\Lambda)
$$

$$
{ }^{\circ} F_{\breve{f}}(b)={ }^{\circ} \phi(b) \quad\left(b \in B^{\prime}\right)
$$

Hence,

$$
F_{f}(b)={ }^{\circ} F_{f}(b)+I_{f}(b)={ }^{\circ} \phi(b)+I_{\phi}(b)=\phi(b) \quad\left(b \in B^{\prime}\right) .
$$

The last line of equalities following directly from the first part of the proof and (9) and (10). 


\section{REFERENCES}

1. J. Arthur, Harmonic analysis of tempered distributions on semisimple Lie groups of rank one, Thesis, Yale, 1970.

2. - Harmonic analysis of the Schwartz space on a reductive Lie group I, preprint.

3. - Harmonic analysis of the Schwartz space on a reductive Lie group II, preprint.

4. D. De George and N. Wallach, Limit formulas for multiplicities in $L^{2}(G / \Gamma) I, I I$, Ann of Math., 107 (1978), 133-150 and 109 (1979), 477-495.

5. Harish-Chandra, Discrete series for semisimple Lie groups II, Acta Math., 116 (1966), 1-111.

6. - Harmonic analysis on real reductive Lie groups I, J. Functional Analysis, 19 (1975), 104-204.

7. - Harmonic analysis on real reductive Lie groups II, Inv. Math., 36 (1976), $1-55$.

8. - Harmonic analysis on real reductive groups, III, Ann. of Math., 104 (1976), 117-201.

9. A. W. Knapp and E. M. Stein, Intertwining operators for semisimple Lie groups, Ann. of Math., 93 (1971), 489-578.

10. D. Milicic, Asymptotic behavior of matrix coefficients of the discrete series, Duke Math. J., 44 (1977), 59-88.

11. Paul J. Sally Jr., and Garth Warner, The Fourier transform on semisimple Lie groups of real rank one, Acta Math., 131 (1973), 1-26.

12. P. C. Trombi, Fourier analysis on semisimple Lie groups of split rank one, Thesis, University of Illinois, 1970.

13. On Harish-Chandra's theory of the Eisenstein integral, Lecture Notes, UCLA.

14. - Asymptotic Expansions of Matrix Coefficients: The Real Rank One Case, J. Functional Analysis, 30 (1978), 83-105.

15. Harmonic analysis of $C^{p}(G: F)(1 \leq \phi<2)$, submitted to the J. Functional Analysis.

16. P. C. Trombi and V. S. Varadarajan, Spherical transforms on semisimple Lie groups, Ann. of Math., 94 (1971), 246-303.

17. - Asymptotic behavior of eigenfunctions on a semisimple Lie group: The discrete spectrum, Acta Math., 129 (1972), 237-280.

18. V. S. Varadarajan, Harmonic Analysis on Real Reductive Groups, Springer-Verlag, Lecture Notes 576, Berlin, New York, 1977.

19. G. Warner, Harmonic Analysis on Semisimple Lie Groups I, II, Springer Verlag, Berlin, New York, 1972.

Received July 10, 1980 and in revised form January 5, 1981.

UNIVERSiTy OF UTAH

Salt Lake City, UT 84112 



\section{PACIFIC JOURNAL OF MATHEMATICS}

\section{EDITORS}

DONALD BABBITT (Managing Editor)

J. DUGUNDJI

University of California

Los Angeles, California 90024

Hugo Rossi

University of Utah

Salt Lake City, UT 84112

C. C. Moore and Arthur Agus

Department of Mathematics

University of Southern California

Los Angeles, California 90007

R. FinN and J. Milgram

Stanford University

Stanford, California 94305

University of California

Berkeley, CA 94720

\section{ASSOCIATE EDITORS}
R. ARNES
E. F. BeCKenBaCH
B. H. NEUMANN
F. WoLF
K. YoshidA

\section{SUPPORTING INSTITUTIONS}

UNIVERSITY OF ARIZONA

UNIVERSITY OF BRITISH COLUMBIA

CALIFORNIA INSTITUTE OF TECHNOLOGY

UNIVERSITY OF CALIFORNIA

MONTANA STATE UNIVERSITY

UNIVERSITY OF NEVADA, RENO

NEW MEXICO STATE UNIVERSITY

OREGON STATE UNIVERSITY
UNIVERSITY OF OREGON

UNIVERSITY OF SOUTHERN CALIFORNIA

STANFORD UNIVERSITY

UNIVERSITY OF HAWAII

UNIVERSITY OF TOKYO

UNIVERSITY OF UTAH

WASHINGTON STATE UNIVERSITY

UNIVERSITY OF WASHINGTON 


\section{Pacific Journal of Mathematics}

\section{Vol. 101, No. $1 \quad$ November, 1982}

Natália Bebiano, On the evaluation of permanents $\ldots \ldots \ldots \ldots \ldots \ldots \ldots \ldots$

David Borwein and Bruce Brigham Watson, Tauberian theorems between

the logarithmic and Abel-type summability methods $\ldots \ldots \ldots \ldots \ldots \ldots 11$

Leo George Chouinard, II, Hermite semigroup rings $\ldots \ldots \ldots \ldots \ldots \ldots \ldots$

Kun-Jen Chung, Remarks on nonlinear contractions $\ldots \ldots \ldots \ldots \ldots \ldots . \ldots 4$

Lawrence Jay Corwin, Representations of division algebras over local

fields. II ......................................... 49

Mahlon M. Day, Left thick to left lumpy—a guided tour $\ldots \ldots \ldots \ldots \ldots 71$

M. Edelstein and Mo Tak Kiang, On ultimately nonexpansive

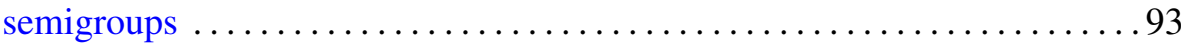

Mary Rodriguez Embry, Semigroups of quasinormal operators . ........ 103

William Goldman and Morris William Hirsch, Polynomial forms on

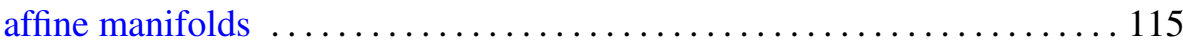

S. Janakiraman and T. Soundararajan, Totally bounded group topologies

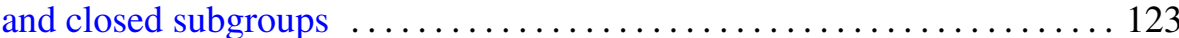

John Rowlay Martin, Lex Gerard Oversteegen and Edward D.

Tymchatyn, Fixed point set of products and cones $\ldots \ldots \ldots \ldots \ldots \ldots 133$

Jan van Mill, A homogeneous Eberlein compact space which is not metrizable ........................................ 141

Steven Paul Plotnick, Embedding homology 3-spheres in $S^{5} \ldots \ldots \ldots \ldots 147$

Norbert Riedel, Classification of the $C^{*}$-algebras associated with minimal rotations

Benedict Seifert, Combinatorial and geometric properties of weight systems of irreducible finite-dimensional representations of simple split Lie algebras over fields of 0 characteristic

James E. Simpson, Dilations on locally convex spaces

Paolo M. Soardi, Schauder bases and fixed points of nonexpansive mappings

Yoshio Tanaka, Point-countable $k$-systems and products of $k$-spaces

Fausto A. Toranzos, The points of local nonconvexity of starshaped sets . . . 209

Lorenzo Traldi, The determinantal ideals of link modules. I . . . . . . . . 215

P. C. Trombi, Invariant harmonic analysis on split rank one groups with applications

Shinji Yamashita, Nonnormal Blaschke quotients 